\title{
Verification of the coupled-momentum method with Womersley's Deformable Wall analytical solution
}

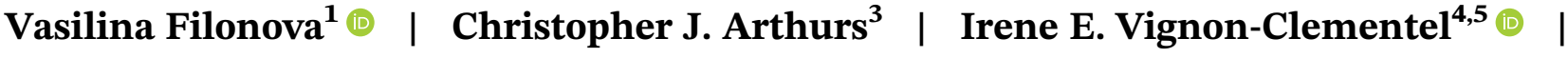 \\ C. Alberto Figueroa ${ }^{1,2,3}$
}

${ }^{1}$ Surgery, University of Michigan, Ann Arbor, MI, USA

${ }^{2}$ Biomedical Engineering, University of Michigan, Ann Arbor, MI, USA

${ }^{3}$ Imaging Sciences and Biomedical Engineering, King's College London, UK

${ }^{4}$ Inria, Paris, France

${ }^{5}$ Sorbonne Université UPMC, Laboratoire Jacques-Louis Lions, Paris, France

\section{Correspondence}

Vasilina Filonova, University of Michigan, North Campus Research Complex, 2800 Plymouth Road, build. 20 - 203W-1A, Ann Arbor, MI 48109.

Email: vfilonov@med.umich.edu

\section{Funding information}

National Institute of Biomedical Imaging and Bioengineering, Grant/Award

Number: grant U01 HL135842

\begin{abstract}
In this paper, we perform a verification study of the Coupled-Momentum Method (CMM), a 3D fluid-structure interaction (FSI) model which uses a thin linear elastic membrane and linear kinematics to describe the mechanical behavior of the vessel wall. The verification of this model is done using Womersley's deformable wall analytical solution for pulsatile flow in a semiinfinite cylindrical vessel. This solution is, under certain premises, the analytical solution of the CMM and can thus be used for model verification. For the numerical solution, we employ an impedance boundary condition to define a reflection-free outflow boundary condition and thus mimic the physics of the analytical solution, which is defined on a semi-infinite domain. We first provide a rigorous derivation of Womersley's deformable wall theory via scale analysis. We then illustrate different characteristics of the analytical solution such as space-time wave periodicity and attenuation. Finally, we present the verification tests comparing the CMM with Womersley's theory.
\end{abstract}

\section{1 | INTRODUCTION}

Blood can be represented as an incompressible fluid whose constitutive behavior is usually approximated, at least in the larger arteries, by a Newtonian model and the incompressible Navier-Stokes equations. Blood velocity, pressure, and propagation of waves within the arterial tree are greatly affected by the deformability of the vessel wall ${ }^{1,2}$. Wave speed and changes in amplitude and phase are dictated by vessel size, viscoelastic behavior, and by blood viscosity. Wave attenuation and dispersion are also observed within the cardiovascular system.

Numerous mathematical formulations have been developed to represent these complex physical phenomena, usually describing oscillatory flow in an idealized tube, rigid or elastic ${ }^{3-8}$. One of such formulations is given by Womersley's analytical velocity profile for oscillatory flow in rigid tubes ${ }^{9}$. This was then extended to the case of compliant arteries by taking into consideration wall deformations and radial components of blood velocity, producing an analytical solution for pulsatile flow in a deformable, axisymmetric, semi-infinite vessel ${ }^{9-13}$. Womersley's deformable wall analytical solution represents an excellent framework to understand some of the governing principles of wave propagation phenomena in the cardiovascular system. It is also a good tool for performing verification studies of mathematical models of blood flow in compliant arteries.

Beyond analytical solutions, computational 3D models have been used extensively to study fluid-structure interactions (FSI) between blood flow and vessel wall motion. Boundary-fitted techniques based on Arbitrary Lagrangian-Eulerian (ALE) formulations ${ }^{14-16}$, non-boundary fitted techniques such as the Immersed Boundary Method ${ }^{17,18}$ or the Fictitious Domain Method ${ }^{19}$, 
or simplified models such as the Coupled-Momentum Method (CMM) ${ }^{20,21}$ have been proposed to describe cardiovascular FSI. These are all complex formulations which require rigorous benchmarking to ensure error-free implementations.

There are several studies that have used analytical solutions for pulsatile flow in compliant vessels for validation and verification of 3D computational FSI models ${ }^{22-26}$. A FSI strategy involving external coupling of ANSYS and CFX solvers for blood flow in a straight elastic tube was compared against a single-frequency Womersley's analytical solution ${ }^{22}$. Verification and validation of a FSI numerical method based on OpenFOAM was described in the work of Kanyanta et al. ${ }^{23}$, comparing numerical results with analytical expressions for pressure wave speed and axial stress perturbations ${ }^{27,28}$, as well as with data from polyurethane mock artery experiments. In the paper of Passerini et al. ${ }^{26}$, validation of the opensource LifeV framework was presented with verification against an analytical solution for wave speed. Ponzini and colleagues ${ }^{24}$ presented an in vivo validation using 2D Phase Contrast Magnetic Resonance Imaging of a Womersley numberbased formula for estimating flow rate in several arteries. In the study of van Geel et al. ${ }^{25}$, an ALE FSI numerical model with viscoelastic walls was compared against Womersley's solution and experimental results, showing good agreement for straight and tapered vessels.

The purpose of this paper is to perform a verification study of the CMM ${ }^{20}$ against a multi-frequency Womersley's deformable wall analytical solution. The CMM is 3D method that considers a monolithic approach for the FSI problem, a thin linear elastic membrane model for the arterial wall, and fixed conforming meshes at the vessel wall-fluid boundary interface. The dynamic coupling between blood and vessel wall was achieved by defining a fictitious body force driving the wall motion, similar to a key assumption in Womersley's deformable wall analytical solution ${ }^{13}$. Womersley's deformable solution can be regarded as the analytical solution for the Coupled-Momentum Method under the following conditions: cylindrical and axisymmetric geometry; linear, periodic flow, and non-reflective outflow boundary conditions. Therefore, it is best suitable for verification.

A key component of this work is the utilization of an impedance boundary condition for the outflow boundary of the computational domain ${ }^{29}$. This approach makes it possible to use a reflection-free outflow boundary which can mimic the physics of the analytical solution, defined on a semi-infinite domain ${ }^{30}$. Furthermore, this approach for outflow boundary condition avoids the direct specification of any of the main solution variables at this boundary, and thus contributes to a more rigorous set-up for the verification of the CMM.

The structure of this paper is as follows. In the methods section, an overview of Womersley's analytical solution for pulsatile flow in a deformable, axisymmetric, semi-infinite cylindrical vessel is provided first in Section $2.1^{13}$. This is complemented by Appendix where the mathematical formulation is recapitulated in concise, non-dimensional form applying scale analysis ${ }^{31}$ to summarize the theory's limitations needed for verification. The CMM 3D formulation is then presented in Section 2.2. Special care was taken to define a non-reflective boundary condition for the numerical domain, using a coupled-multidomain method ${ }^{29}$. In the results section, first in Sections 3.1-3.2 a multi-frequency Womersley deformable solution is determined, in order to represent flow, pressure and wall motion in an idealized model of a human common carotid artery. Finally, in Section 3.3 numerical solutions of the CMM were compared against the analytical solution. Special care was taken in the definition of the problem parameters to ensure that solutions are both physiologically realistic and compatible with the assumptions of Womersley's theory.

\section{2 | METHODS}

In this section we carefully describe the assumptions and governing equations leading to Womersley's theory and to the CMM method. We focus on the main assumptions relevant for the comparison of the methods. For example, Womersley's theory is described on a semi-infinite cylindrical vessel using 2D axisymmetric assumptions and linear fluid-solid interactions. Conversely, the CMM is a numerical formulation capable of dealing with 3D geometries and nonlinear flows on finite domains. However, under certain assumptions the CMM can be reduced to the Womersley's deformable wall formulation. These assumptions include: axisymmetric linear flow, straight elastic vessels with thin walls, a total wall surface traction used to define a wall body force, and no wave-reflections. The latter assumption is enforced in the finite numerical domain via an outflow impedance boundary condition.

\section{1 | Womersley theory for blood flow in a deformable vessel}

The Womersley's theory describes the axisymmetric motion of blood when subjected to a periodic pressure gradient in a straight elastic vessel. Under a linear assumption, the pumping action of the heart results in a pressure gradient $k_{\text {total }}$, 
which can be decomposed into a constant component $k_{s}$ producing a steady forward flow, and an oscillatory component $k$ with zero net flow over the cardiac cycle ${ }^{32}$ :

$$
\frac{\partial p_{\text {total }}}{\partial z}=k_{\text {total }}(r, z, t)=k_{s}+k(r, z, t)
$$

where $p_{\text {total }}$ is a total pressure field with steady and oscillatory contributions; $r$ and $z$ are the radial and longitudinal coordinates of the tube, respectively, and $t$ is a time variable.

The total velocity field in the longitudinal and radial directions can be written as:

$$
\begin{gathered}
\text { Longitudinal }: w_{\text {total }}=w_{s}(r)+w(r, z, t)=\frac{k_{s}}{4 \mu}\left(r^{2}-R^{2}\right)+w(r, z, t), \\
\text { Radial }: u_{\text {total }}=u_{s}(r)+u(r, z, t)=u(r, z, t)
\end{gathered}
$$

where $w$ and $u$ are the oscillatory components of the velocity field. The steady Poiseuille longitudinal component $w_{s}$ is added to $w$ to reconstruct the total velocity profile. In the radial direction, the steady component of the velocity $u_{s}(r)$ is identically zero. Here, $R$ is the vessel radius and $\mu$ is the blood dynamic viscosity. Similarly, the total pressure field $p_{\text {total }}$ can be decomposed in terms of its steady, $p_{s}$, and pulsatile, $p$, components as:

$$
p_{\text {total }}=p_{s}(z)+p(r, z, t)=p_{0}+k_{s} z+p(r, z, t)
$$

where $p_{0}$ is the mean temporal pressure at the inlet of the vessel $(z=0)$.

The complete derivation of Womersley's governing equations for a freely-moving elastic, cylindrical and semi-infinite vessel ${ }^{13}$ is presented next. The presented derivation refers only to the oscillatory components of the velocity and pressure field $\left(w, u\right.$, and $p$ ). A scale analysis (order of magnitude analysis) ${ }^{31}$ of the governing equations is used to understand the validity limits of the simplifying assumptions of theory. The following scaling rules were considered for blood flow and vessel wall variables:

$$
\begin{gathered}
r=R r^{\prime}, z=z^{\prime} \frac{c}{\omega}, t=\frac{t^{\prime}}{\omega}, u=\varepsilon c u^{\prime}, w=\delta c w^{\prime}, p=\gamma \rho \delta c^{2} p^{\prime}, \\
\xi=\frac{\varepsilon c}{\omega} \xi^{\prime}, \zeta=\frac{\delta c}{\omega} \zeta^{\prime},
\end{gathered}
$$

where $\omega$ is the angular frequency of oscillations, $c$ is the wave speed, $\rho$ is the blood density, and $\delta, \varepsilon, \gamma$ are nondimensional scale parameters for the longitudinal velocity, radial velocity, and pressure, respectively. $\xi$ and $\zeta$ represent the oscillatory radial and longitudinal displacements, respectively. In equations (5) and (6) all non-dimensional variables, denoted by primes, are assumed to be of the same order $\sim \mathcal{O}(1)$.

\subsection{1 | Blood flow equations}

In a cylindrical system of coordinates and using the non-dimensional form (5), the mass balance equation and NavierStokes equations for momentum balance in the radial and longitudinal directions are given by:

$$
\begin{gathered}
\frac{\partial u^{\prime}}{\partial r^{\prime}}+\frac{u^{\prime}}{r^{\prime}}+\frac{\partial w^{\prime}}{\partial z^{\prime}}=0, \\
\frac{\partial u^{\prime}}{\partial t^{\prime}}+\delta\left(u^{\prime} \frac{\partial u^{\prime}}{\partial r^{\prime}}+w^{\prime} \frac{\partial u^{\prime}}{\partial z^{\prime}}\right)=-\frac{\gamma}{\beta^{2}} \frac{\partial p^{\prime}}{\partial r^{\prime}}+\frac{1}{\alpha^{2}}\left[\frac{\partial^{2} u^{\prime}}{\partial r^{\prime 2}}+\frac{1}{r^{\prime}} \frac{\partial u^{\prime}}{\partial r^{\prime}}-\frac{u^{\prime}}{r^{\prime 2}}+\beta^{2} \frac{\partial^{2} u^{\prime}}{\partial z^{\prime 2}}\right],
\end{gathered}
$$




$$
\frac{\partial w^{\prime}}{\partial t^{\prime}}+\delta\left(u^{\prime} \frac{\partial w^{\prime}}{\partial r^{\prime}}+w^{\prime} \frac{\partial w^{\prime}}{\partial z^{\prime}}\right)=-\gamma \frac{\partial p^{\prime}}{\partial z^{\prime}}+\frac{1}{\alpha^{2}}\left[\frac{\partial^{2} w^{\prime}}{\partial r^{\prime 2}}+\frac{1}{r^{\prime}} \frac{\partial w^{\prime}}{\partial r^{\prime}}+\beta^{2} \frac{\partial^{2} w^{\prime}}{\partial z^{\prime 2}}\right]
$$

where the non-dimensional parameter $\alpha=R \sqrt{\omega / \nu}$ is the Womersley number, and $\nu=\mu / \rho$ is the blood kinematic viscosity.

The Navier-Stokes equations (8), (9) can be significantly simplified under the long-wave approximation assumptions, namely: 1) the characteristic flow wavelength $\lambda=2 \pi c / \omega$ is much larger than the vessel radius $R$; 2) the wave speed $c$ is much larger than blood velocity components. From the first condition, it follows that the non-dimensional scale parameter $\beta=\omega R / c \ll<1$; and from the second condition the velocity scale parameters introduced in equation (5) should be small: $\varepsilon, \delta \ll 1$. Moreover, from equation (7) the following relations apply to scale parameters, oscillatory velocities, and coordinates:

$$
\frac{\varepsilon}{\delta}=\beta \ll 1, \frac{u}{w}=\beta \frac{u^{\prime}}{w^{\prime}} \ll 1, \frac{r}{z}=\beta \frac{r^{\prime}}{z^{\prime}} \ll 1
$$

All non-dimensional variables in equations (8), (9) are $\mathcal{O}(1)$ due to the scaling rule. The non-linear term (advective inertial forces) are $\mathcal{O}(\delta)$ and the viscous stress axial terms are $\mathcal{O}\left(\beta^{2}\right)$ and can thus be neglected. The simplified momentum balance equations become linear and therefore amenable for superposition of solutions in a harmonic wave form.

Boundary conditions for the fluid problem include: no-slip at the fluid-solid interface (e.g. matching velocities of fluid and solid at the wall), imposed oscillatory velocities $\hat{u}$ and $\hat{w}$ at the tube inlet, and finite velocity at the vessel centerline.

Remark 1 When performing the verification study of the CMM, it is important to estimate the contribution of the non-linear advective term present in the numerical solution but absent from the analytical solution. The nondimensional scale parameter $\delta$ can be estimated from (5) as $\delta \approx \bar{w} / c$, where $\bar{w}(z, t)=\langle w(r, z, t)\rangle_{R}$ is the averaged longitudinal velocity over the radius. The oscillatory flow rate can be written as $q(z, t)=\pi R^{2} \bar{w}(z, t)$. The upper bound of the non-linear advective scale parameter of the numerical solution $\delta^{\text {num }}$ can be estimated from the maximum oscillatory flow at the inlet $q^{\text {inp }}$ as

$$
\delta^{\text {num }}=\frac{\max _{t \in[0, T]} q_{\text {total }}^{\text {inp }}-q_{s}}{\pi R^{2} c^{\text {num }}},
$$

where $q_{\text {total }}^{\text {inp }}-q_{s}=q(z=0, t)=q^{\text {inp }}$, and $c^{\text {num }}$ is the reconstructed wave speed from the numerical solution.

In addition, to check satisfaction of $u / c \ll 1$, one may estimate $\varepsilon \approx \delta \beta$, utilizing relation (10).

Remark 2 Radial and longitudinal pressure gradients should not be neglected to obtain non-trivial velocity solutions. These terms, scaled by $\gamma$ and $\gamma / \beta^{2}$ in equations (8), (9), are preserved since they do not affect the linearity of the equations regardless of their order, but they do affect the solution.

\subsection{2 | Vessel wall equations}

The vessel wall equations of motion are written in the context of linear elasticity and thin-walled tube theories, assuming small radial deformations. Cauchy's equation of motion is $\rho^{w} \partial^{2} \boldsymbol{s} / \partial t^{2}=\mathbf{F}+\nabla \cdot \boldsymbol{T}$, where $\rho^{w}$ is the wall density, $\mathbf{F}$ a body force per unit volume, $\boldsymbol{T}$ the Cauchy stress tensor for the vessel wall, and $\varsigma=[\xi, 0, \zeta]^{T}$ is the oscillatory displacement vector in cylindrical coordinates.

The thin-wall assumption states:

$$
h<<,|\xi|<<R
$$

where $h$ is the vessel wall thickness. The solid domain is modeled as a membrane and thus a 2D interface with the lateral boundary of the fluid domain. Relations (12) imply small radial deformations and thus the average radial 
coordinate of the vessel wall is $r \approx R$. Therefore, a no-slip kinematic boundary condition at the fluid-solid interface, $r=R$, is simply $\left.u\right|_{r=R}=\partial \xi / \partial t,\left.w\right|_{r=R}=\partial \zeta / \partial t$.

Further exploiting the thin-wall assumption, Womersley defined radial and longitudinal components of a fictitious body force $\mathbf{F}$ driving the dynamics of the membrane from the pressure $p$ and shear stresses $\tau$ acting on the lateral fluid boundary as:

$$
F_{r}=\frac{\left.p\right|_{r=R}}{h}, F_{z}=-\frac{\tau}{h}=-\left.\frac{\rho \nu}{h}\left(\frac{\partial u}{\partial z}+\frac{\partial w}{\partial r}\right)\right|_{r=R} .
$$

The stress tensor $\boldsymbol{T}$ is defined by considering two stress states: internal pressurization with no axial strain, and axial force with no internal pressure. The linear superposition of these states yields the following circumferential and longitudinal components of the stress tensor ${ }^{13}$ :

$$
T_{\theta \theta}=B\left(\frac{\xi}{R}+\sigma \frac{\partial \zeta}{\partial z}\right), T_{z z}=B\left(\sigma \frac{\xi}{R}+\frac{\partial \zeta}{\partial z}\right)
$$

where $B=E /\left(1-\sigma^{2}\right), E$ is the Young's modulus of the vessel wall, and $\sigma$ is its Poisson's ratio.

Using equations (5), (6), (12), (13) and neglecting smaller terms in the divergence of the stress tensor, $(\nabla \cdot \boldsymbol{T})_{r} \approx-\left.\left(T_{\theta \theta} / r\right)\right|_{r=R}$ and $(\nabla \cdot \boldsymbol{T})_{z} \approx \partial T_{z z} / \partial z$, we obtain the radial and longitudinal equations of motion for the vessel wall in non-dimensional form:

$$
\begin{gathered}
\beta^{2} \frac{\partial^{2} \xi^{\prime}}{\partial t^{\prime 2}}=\left.\gamma \frac{\rho R}{\rho^{w} h} p^{\prime}\right|_{r^{\prime}=1}-\frac{B}{\rho^{w} \mathcal{c}^{2}}\left(\xi^{\prime}+\sigma \frac{\partial \zeta^{\prime}}{\partial z^{\prime}}\right), \\
\frac{\partial^{2} \zeta^{\prime}}{\partial t^{\prime 2}}=-\left.\frac{\rho \nu}{\rho^{w} h \omega R}\left(\beta^{2} \frac{\partial u^{\prime}}{\partial z^{\prime}}+\frac{\partial w^{\prime}}{\partial r^{\prime}}\right)\right|_{r^{\prime}=1}+\frac{B}{\rho^{w} c^{2}}\left(\sigma \frac{\partial \xi^{\prime}}{\partial z^{\prime}}+\frac{\partial^{2} \zeta^{\prime}}{\partial z^{\prime 2}}\right) .
\end{gathered}
$$

The non-dimensional coefficient $B /\left(\rho^{w} c^{2}\right)>1$ because the velocity of shear waves in the material of the tube is greater than the pulse wave velocity ${ }^{13}$. Using long-wave approximation, $\beta^{2} \ll 1$, equation (15) can be reduced to a radial equilibrium equation. Similarly, the term $\partial u^{\prime} / \partial z^{\prime}$ in equation (16) can be neglected.

Remark 3 The small deformation assumption in equation (12) does not apply to the axial direction, in which the longitudinal deformations can be finite. Womersley developed an extension of the theory ${ }^{11}$ where an additional longitudinal wall motion elastic constraint was introduced to reduce axial wall deformations, see Remark 5.

\subsection{3 | Summary of fluid-solid equations and solutions}

The resulting non-dimensional, linear system of second order differential equations for the fluid-solid problem is summarized in Table 1. The solution in harmonics waves is completely derived in Appendix and summarized in Table 2. The wave speed $c$ is defined by frequency equations as described in Appendix. Initial conditions for velocity, pressure, and wall displacements must be provided. Lastly, velocities at the center of the vessel are assumed to be finite, Table 1 (C).

Remark 4 The radial displacement $\xi$ is related to oscillatory flow rate $q$, Table 2, so that the radial wall deformations $\xi / R$ can be shown to be of order $\delta / 2$ :

$$
\xi(z, t)=\frac{q(z, t)}{2 \pi R c}=\frac{R \bar{w}(z, t)}{2 c}, \frac{\xi}{R}=\frac{\bar{w}}{2 c} \approx \frac{\delta}{2} .
$$

Thus, the small deformation part of the thin-wall approximation (12) is linked to the parameter $\delta$ which itself is related to the long-wave approximation. Therefore, provided that $h \ll R$, the order of the parameter $\delta$ represents a validity check for both these assumptions, and thus for the applicability of Womersley's theory. 
T A B L E 1 Womersley's deformable wall theory: Governing equations, assumptions and boundary conditions

\section{Non-dimensional system of governing equations for oscillatory $p^{\prime}, u^{\prime}, w^{\prime}, \xi^{\prime}, \zeta^{\prime}$}

\section{(A): Fluid}

$$
\begin{aligned}
& \frac{\partial u^{\prime}}{\partial r^{\prime}}+\frac{u^{\prime}}{r^{\prime}}+\frac{\partial w^{\prime}}{\partial z^{\prime}}=0 \\
& \frac{\partial u^{\prime}}{\partial t^{\prime}}=-\frac{\gamma}{\beta^{2}} \frac{\partial p^{\prime}}{\partial r^{\prime}}+\frac{1}{\alpha^{2}}\left[\frac{\partial^{2} u^{\prime}}{\partial r^{\prime 2}}+\frac{1}{r^{\prime}} \frac{\partial u^{\prime}}{\partial r^{\prime}}-\frac{u^{\prime}}{r^{\prime 2}}\right] \\
& \frac{\partial w^{\prime}}{\partial t^{\prime}}=-\gamma \frac{\partial p^{\prime}}{\partial z^{\prime}}+\frac{1}{\alpha^{2}}\left[\frac{\partial^{2} w^{\prime}}{\partial r^{\prime 2}}+\frac{1}{r^{\prime}} \frac{\partial w^{\prime}}{\partial r^{\prime}}\right]
\end{aligned}
$$

(C): Boundary conditions $\left.u^{\prime}\right|_{z^{\prime}=0}=\hat{u}\left(r^{\prime}, t^{\prime}\right),\left.w^{\prime}\right|_{z^{\prime}=0}=\hat{w}\left(r^{\prime}, t^{\prime}\right)$

$\left|u^{\prime},\right|_{r^{\prime}=0}<+\infty,\left|w^{\prime},\right|_{r^{\prime}=0}<+\infty$

(D): Long-wave approximation

$u / c \ll 1, w / c \ll 1, \beta \ll 1$

(F): Scaling rule

\section{(B): Solid}

$$
\begin{aligned}
& 0=\left.\gamma \frac{\rho R}{\rho^{w} h} p^{\prime}\right|_{r^{\prime}=1}-\frac{B}{\rho^{w} c^{2}}\left(\xi^{\prime}+\sigma \frac{\partial \zeta^{\prime}}{\partial z^{\prime}}\right) \\
& \frac{\partial^{2} \zeta^{\prime}}{\partial t^{\prime 2}}=-\left.\frac{\rho \nu}{\rho^{w} h \omega R}\left(\frac{\partial w^{\prime}}{\partial r^{\prime}}\right)\right|_{r^{\prime}=1}+\frac{B}{\rho^{w} c^{2}}\left(\sigma \frac{\partial \xi^{\prime}}{\partial z^{\prime}}+\frac{\partial^{2} \zeta^{\prime}}{\partial z^{\prime 2}}\right)
\end{aligned}
$$

$$
\begin{aligned}
& r=R r^{\prime}, z=z^{\prime} c / \omega, t=t^{\prime} / \omega, \\
& u=\varepsilon c u^{\prime}, w=\delta c w^{\prime}, p=\gamma \rho \delta c^{2} p^{\prime} \\
& \delta \ll 1, \varepsilon / \delta=\beta=\omega R / c \ll 1
\end{aligned}
$$

$$
\left.u^{\prime}\right|_{r^{\prime}=1}=\left.\left(\frac{\partial \xi^{\prime}}{\partial t^{\prime}}\right)\right|_{r^{\prime}=1},\left.w^{\prime}\right|_{r^{\prime}=1}=\left.\left(\frac{\partial \zeta^{\prime}}{\partial t^{\prime}}\right)\right|_{r^{\prime}=1}
$$

\section{(E): Thin-wall assumption}

$$
h \ll R,|\xi| \ll R
$$

$$
\xi=\xi^{\prime} \varepsilon c / \omega, \zeta=\zeta^{\prime} \delta c / \omega
$$

\section{(G): Parameters}

$\omega, R, \nu, \rho, \alpha=R \sqrt{\omega / \nu}$

$$
\rho^{w}, h, B=E /\left(1-\sigma^{2}\right)
$$

\section{Radial oscillatory velocity}

$u(r, z, t)=i \frac{H \omega R}{2 \rho c^{2}}\left(\frac{r}{R}-M \frac{2 J_{1}(\Lambda r / R)}{\Lambda J_{0}(\Lambda)}\right) \exp (i \omega(t-z / c))$

\section{Axial oscillatory velocity}

$w(r, z, t)=\frac{H}{\rho c}\left(1-M \frac{J_{0}(\Lambda r / R)}{J_{0}(\Lambda)}\right) \exp (i \omega(t-z / c))$

\section{Oscillatory flow and pressure}

$q(z, t)=\frac{\pi R^{2} H}{\rho c}(1-M g) \exp (i \omega(t-z / c)), p(z, t)=H \exp (i \omega(t-z / c))$

\section{Wave speed relations}

$c^{2}=c_{0}^{2} \frac{2}{\left(1-\sigma^{2}\right) v}, c_{R}=\left(\operatorname{Re}\left\{c^{-1}\right\}\right)^{-1}, c_{I}=\left(\operatorname{Im}\left\{c^{-1}\right\}\right)^{-1}$

Solve for $v$ : $[1-g]\left(1-\sigma^{2}\right) v^{2}-\left[2+\frac{\rho^{w} h}{\rho R}(1-g)+g\left(\frac{1}{2}-2 \sigma\right)\right] v+g+2 \frac{\rho^{w} h}{\rho R}=0$

\section{Parameters}

$H, \omega, R, h, \nu, \rho, \rho^{w}, \sigma, E, \Lambda=i^{3 / 2} \alpha, c_{0}^{2}=\frac{E h}{2 \rho R}, g=\frac{2 J_{1}(\Lambda)}{\Lambda J_{0}(\Lambda)}, M=\frac{2+v(2 \sigma-1)}{v(2 \sigma-g)}$

\section{Radial wall displacement}

$\xi(z, t)=\frac{R H}{2 \rho c^{2}}(1-M g) \exp (i \omega(t-z / c))$

\section{Axial wall displacement}

$\zeta(z, t)=\frac{i H}{\rho c \omega}(M-1) \exp (i \omega(t-z / c))$
TABLE 2 Dimensional (complex) single-frequency solution for Womersley's deformable wall theory (see Appendix)

Remark 5 The solutions in Table 2 can be reduced to a more physiologically relevant case of longitudinally tethered vessels ${ }^{11}$, i.e. $\zeta=0$ which yields $M=1$ and wave speed $c=c_{0} \sqrt{(1-g) /\left(1-\sigma^{2}\right)}$. The axial velocity $w$ in this case is the same as for the rigid wall case ${ }^{9}$ and is the most known Womersley's result.In 1D theories for blood flow in elastic vessels, this Womersley's velocity profile is often assumed and used to enhance a theory with a friction model thus implying longitudinally tethering of vessel walls ${ }^{8,33,34}$. There radial velocity and wave speed can be also derived using a perturbation method for linearization ${ }^{8}$.

\subsection{4 | Analytical impedance}

A key challenge in verification of CMM versus Womersley's solution is that the analytical solution is defined on a semiinfinite domain, while the numerical solution is defined on a finite domain. To circumvent this issue, a reflection-free 
impedance function will be used as outflow boundary condition for the computational domain. This impedance function can be derived from the analytical solution for flow and pressure at any axial location of the vessel. Furthermore, this approach avoids the direct specification of any solution variables as outflow boundary conditions, thereby rendering a more rigorous testbed for the verification analysis.

The impedance is a measure of the opposition to oscillatory flow ${ }^{5}$. In the frequency domain, impedance is defined as the ratio of pressure to flow rate for each frequency mode: $Z_{n}\left(z, \omega_{n}\right)=P_{n}\left(z, \omega_{n}\right) / Q_{n}\left(z, \omega_{n}\right)$. For Womersley's solution, since there are no wave reflections, this impedance becomes the characteristic impedance, a function solely of the vessel and fluid properties, and thus position-independent:

$$
Z_{n}\left(\omega_{n}\right)=\frac{\rho c_{n}}{\pi R^{2}\left(1-M_{n} g_{n}\right)}, n \neq 0
$$

For $n=0$, the impedance is the ratio of steady pressure to steady flow $Z_{0}(z)=p_{s}(z) / q_{s}$, a quantity that depends on the axial position: $Z_{0}(z)=-8 \mu\left(p_{0}+k_{s} z\right) / k_{s} \pi R^{4}$. In the time domain, the impedance function $\bar{z}(z, t)$, is obtained via the inverse Fourier transform of $Z_{n}\left(z, \omega_{n}\right)$ as:

$$
\bar{z}(z, t)=Z_{0}(z)+2 \operatorname{Re}\left\{\sum_{n=1}^{N / 2} Z_{n}\left(z, \omega_{n}\right) \exp \left(i \omega_{n} t\right)\right\} .
$$

The total pressure-flow relationship in time domain can be written as a convolution integral of impedance and flow as follows:

$$
p_{\text {total }}(z, t)=\frac{1}{T} \int_{t-T}^{t} q_{t o t a l}\left(z, t_{1}\right) \bar{z}\left(z, t-t_{1}\right) d t_{1}
$$

Here, pressure at a given time depends not only on the flow rate at that time instant but also on the flow rate and pressure at previous times. Such history-dependent behavior can be observed in the cardiovascular system due to blood inertia, arterial distensibility, pulse wave propagation, reflection, etc ${ }^{29}$.

\section{2 | Coupled-momentum method formulation}

The CMM is implemented in the open-source software CRIMSON ${ }^{35}$. The Coupled-Momentum Method formulation for fluid-structure interactions ${ }^{20,36}$ is based on a stabilized finite element formulation for the Navier-Stokes equations and has been used to solve large-scale cardiovascular flows in 3D subject-specific domains ${ }^{21,35}$. Inspired by Womersley's deformable wall theory, the method embeds the linear elasto-dynamic response of the wall into a single variational form for the FSI system via a fictitious body force driving the motion of the membrane. The fictitious body force is defined from the total traction (e.g., pressure and wall shear stress) at the fluid-solid interface. This results in a monolithic method whereby the degrees-of-freedom of the vessel wall and the fluid boundary are identical, thus naturally satisfying the no-slip condition. The membrane displacements are obtained by consistent time integration of the fluid velocities and accelerations at the interface. Lastly, a linearized kinematics Eulerian approach is adopted for the coupled problem, and thus fluid-solid grids are kept fixed. The solution of the resulting systems of equations is done via iterative GMRES algorithms.

\subsection{1 | Strong form of fluid and solid equations}

Blood flow in the large vessels of the cardiovascular system can be approximated as the flow of an incompressible Newtonian fluid in a spatial (Eulerian) domain $\Omega$ and time $(0, T)$. The boundary $\Gamma$ of fluid domain $\Omega$ can be divided into three different nonoverlapping partitions such that $\Gamma=\partial \Omega=\Gamma_{g} \cup \Gamma_{h} \cup \Gamma_{s}$. The fluid continuity and momentum balance equations with boundary and initial conditions in the strong form on $(\mathbf{x}, t) \in \Omega \times(0, T)$ are: 


$$
\begin{gathered}
\nabla \cdot \mathbf{v}=0, \rho \mathbf{v}_{, t}+\rho \mathbf{v} \cdot \nabla \mathbf{v}=-\nabla p_{\text {total }}+\nabla \cdot \boldsymbol{\tau} \\
\left.\mathbf{v}\right|_{\Gamma_{g}}=\mathbf{g},\left.\mathbf{v}\right|_{t=0}=\mathbf{v}^{0},\left.\mathbf{t}_{\mathbf{n}}\right|_{\Gamma_{h}}=\left(-p_{\text {total }} I+\tau\right) \mathbf{n}=\mathbf{h}^{f},\left.\mathbf{t}_{\mathbf{n}}\right|_{\Gamma_{S}}=\mathbf{t}^{f}
\end{gathered}
$$

Here, $\mathbf{v}$ represents the blood velocity vector, and $\boldsymbol{\tau}$ is the viscous stress tensor defined as $\boldsymbol{\tau}=\mu\left(\nabla \mathbf{v}+(\nabla \mathbf{v})^{T}\right)$. Body forces are omitted here for the sake of simplicity. The initial velocity $\mathbf{v}^{0}$ is divergent-free. $\Gamma_{g}$ represents the Dirichlet boundary where a given velocity field $\mathbf{g}$ is prescribed (typically the inflow face). $\Gamma_{S}$ is the fluid-solid interface boundary with a prescribed traction $\mathbf{t}^{f}$, and $\Gamma_{\mathbf{h}}$ is a boundary on which a traction $\mathbf{h}^{f}$ is imposed, typically an outflow face, with $\mathbf{n}$ being the face normal.

The vessel wall mechanics are approximated using a thin-walled structure assumption, and therefore the solid domain $\Omega^{s}$ is topologically defined by the same surface as lateral boundary of fluid domain $\Gamma_{s}$. The edges $\partial \Gamma_{g}$ and $\partial \Gamma_{\mathrm{h}}$ represent the parts of the boundary $\Omega^{s}$ where the essential $\mathbf{g}^{s}$ and natural $\mathbf{h}^{s}$ boundary conditions are prescribed. The elastodynamic equations with boundary and initial conditions for the vessel wall can be written as follows:

$$
\begin{gathered}
\rho^{s} \mathbf{u}_{, t t}=\nabla \cdot \boldsymbol{\sigma}^{s}+\mathbf{b}^{s}, \\
\left.\mathbf{u}\right|_{\partial \Gamma_{\mathrm{g}}}=\mathbf{g}^{s},\left.\mathbf{u}\right|_{t=0}=\mathbf{u}^{0},\left.\mathbf{u}_{, t}\right|_{t=0}=\mathbf{u}_{, t}^{0},\left.\mathbf{t}_{n}\right|_{\partial \Gamma_{\mathrm{h}}}=\sigma^{s} \mathbf{n}=\mathbf{h}^{s},
\end{gathered}
$$

where $\mathbf{u}$ is the total wall displacement vector, $\rho^{s}$ is the density of vessel wall, $\mathbf{b}^{s}$ is a body force per unit volume, $\boldsymbol{\sigma}^{s}(\mathbf{u})$ is the vessel wall Cauchy stress tensor, and $\mathbf{u}^{0}$ and $\mathbf{u}_{, t}^{0}$ are the given initial displacement and velocity, respectively. $\mathbf{h}^{s}$ is a traction condition prescribed on the boundary of $\Gamma_{\mathrm{h}}$.

There are two conditions on $\Gamma_{S}$ coupling the fluid and solid problems, inspired by Womersley's deformable wall theory: (i) no-slip condition $\left.\mathbf{v}\right|_{\Gamma_{S}}=\mathbf{u}_{, t}$ and (ii) surface traction equality. The surface traction $\mathbf{t}^{f}$ acting on the fluid lateral boundary due to interaction with the solid is equal and opposed to the surface traction $\mathbf{t}^{s}$ acting on the vessel wall due to the fluid: $\mathbf{t}^{f}=-\mathbf{t}^{s}$. Using a thin-wall approximation, the surface traction $\mathbf{t}^{s}$ can be used to define a fictitious body force $\mathbf{b}^{s}$ acting on the solid domain. Thus, on $\Gamma_{S}$ we have: $\mathbf{b}^{s}=-\mathbf{t}^{f} / h$, similar to equations (13).

\subsection{2 | Weak form equations and impedance outflow boundary condition}

For the weak form equations solid domain $\Omega^{s}$ is mapped on surface $\Gamma_{S}$ such as: $\int_{\Omega^{s}}(\cdot) d \mathbf{x}=h \int_{\Gamma_{S}}(\cdot) d s$ and $\int_{\Gamma_{h}^{s}}(\cdot) d s=h \int_{\partial \Gamma_{h}}(\cdot) d l$. Thus, the weak form for the FSI problem is:

$$
\begin{aligned}
& \int_{\Omega}\left\{\mathbf{w} \cdot\left(\rho \mathbf{v}_{, t}+\rho \mathbf{v} \cdot \nabla \mathbf{v}\right)+\nabla \mathbf{w}:\left(-p_{\text {total }} \mathbf{I}+\boldsymbol{\tau}\right)-\nabla q \cdot \mathbf{v}\right\} d \mathbf{x} \\
& -\int_{\Gamma_{h}} \mathbf{w} \cdot \mathbf{h}^{f} d s+\int_{\Gamma} q \mathbf{v}_{n} d s+h \int_{\Gamma_{S}}\left\{\rho^{s} \mathbf{w} \cdot \mathbf{v}_{, t}+\nabla \mathbf{w}: \boldsymbol{\sigma}^{s}\right\} d s-h \int_{\partial \Gamma_{h}} \mathbf{w} \cdot \mathbf{h}^{s} d l=0,
\end{aligned}
$$

where $\mathbf{w}$ and $\mathrm{q}$ are weighting functions for the momentum and mass balance, respectively.

The traction $\mathbf{h}^{f}$ is defined according to the coupled-multidomain method ${ }^{29}$ using operators $M_{m}, H_{m}$ that represent the behavior of mathematical models of flow distal to the boundary $\Gamma_{h}$ :

$$
\int_{\Gamma_{h}} \mathbf{w} \cdot \mathbf{h}^{f} d s \approx \int_{\Gamma_{h}} \mathbf{w} \cdot\left(M_{m}\left(\mathbf{v}, p_{\text {total }}\right)+H_{m}\right) \mathbf{n} d s
$$

Here, the operators $M_{m}, H_{m}$ are explicitly defined from the physics of pulsatile flow in elastic tube via the impedance function $\bar{z}(z, t)$ as follows:

$$
\begin{gathered}
\int_{\Gamma_{\mathrm{h}}} \mathbf{w} \cdot\left(M_{m}\left(\mathbf{v}, p_{\text {total }}\right)+H_{m}\right) \mathbf{n} d s \approx-\int_{\Gamma_{\mathrm{h}}} \mathbf{w} \cdot \mathbf{n}\left(\frac{1}{T} \int_{t-T}^{t} \bar{z}\left(z, t-t_{1}\right) q\left(t_{1}\right) d t_{1}\right) d s \\
=-\int_{\Gamma_{\mathrm{h}}} \mathbf{w} \cdot \mathbf{n}\left(\frac{1}{T} \int_{t-T}^{t} \bar{z}\left(z, t-t_{1}\right)\left[\int_{\Gamma_{\mathrm{h}}} v_{n}\left(t_{1}\right) d s\right] d t_{1}\right) d s
\end{gathered}
$$


Equation (27) represents an implicitly coupled boundary condition because only the impedance function is given at the boundary $\Gamma_{h}$, whilst pressure and velocity remain unknown solution variables.

\section{3 | RESULTS}

This section is divided into three parts. In the first subsection, the problem material and geometrical parameters are presented and discussed in terms of theory validity. In the second subsection, the analytical solution and its key physical properties (periodicity, attenuation) are demonstrated in a semi-infinite domain. Finally, the third subsection presents the verification study of the CMM numerical results versus Womersley's analytical solution in a finitesize domain.

\section{1 | Geometric and material parameters}

A cylindrical vessel with typical dimensions, material properties, and flow and pressure conditions corresponding to a human common carotid artery are considered here. The input blood flow is taken from the common carotid flow data $q_{\text {total }}^{\text {inp }}(t)$ used in previous studies ${ }^{29}$. Table 3 summarizes material and hemodynamic parameters for the problem. The flow is laminar as defined by a relatively low Reynolds number.

The inlet flow data, Figure 1 (left), is approximated using a 10-term Fourier reconstruction. Table 4 lists the Fourier coefficients, $Q_{n}^{\text {inp }}$. The total input pressure gradient, see Figure 1 (right), $\partial p_{\text {total }} / \partial z=k_{\text {total }}=k_{s}+k$ can be calculated from the analytical solution, having $k_{s}=-8 \mu q_{s} /\left(\pi R^{4}\right)$ and $k(t)=\sum_{n=1}^{N=9} i \omega_{n} H_{n}^{i n p} \exp \left(i \omega_{n} t\right)$, where $q_{s}=Q_{0}^{i n p}$ and $H_{n}^{i n p}$ is a function of $Q_{n}^{\text {inp }}$, given by relation (A17).

\subsection{1 | Validity of linearity and long-wave approximation assumptions}

For the linearity assumptions of the analytical solution to hold, the velocity scale parameters must be $\delta, \varepsilon \ll 1$, equation (5). Furthermore, the long-wave approximation demands that $\beta \ll 1$, equation (10). These parameters are evaluated at the leading frequency $\omega_{1}=2 \pi / T$, Table 5. The Womersley number $\alpha_{1}=3.585$ is slightly below reported physiological values $4.4-5 .{ }^{24,37}$ The real part of the leading frequency of the wave speed $c_{R 1}=643.519 \mathrm{~cm} / \mathrm{s}$ is on the lower bound of the 6.4-10.2 m/s reported for human common carotid artery ${ }^{38}$. The leading frequency spatial wavelength is $\lambda_{1}=707.871 \mathrm{~cm}$, consistent with a long-wave approximation given the radius of the vessel. Using the wave speed $c_{R 1}$, the scale parameters $\delta_{1}, \beta_{1}=\omega_{1} R / c_{R 1}$ and $\varepsilon_{1}=\delta_{1} \beta_{1}$ are evaluated in Table 5. Here, $\delta_{1}=\max _{t \in[0, T]} \bar{w}^{\text {inp }} / c_{R 1}$, where $\max _{t \in[0, T]} \bar{w}^{i n p}=\max _{t \in[0, T]} q_{t o t a l}^{\text {inp }} /\left(\pi R^{2}\right)-w_{s}$. All scale parameters are shown to be small under the set of material and

TABLE 3 Material and hemodynamic parameters for the analytical solution

\begin{tabular}{|llll}
\hline Material and hemodynamic parameters & & \\
\hline Vessel radius $R$ & $0.3 \mathrm{~cm}$ & Mean flow $q_{s}$ & $6.5 \mathrm{~cm}^{3} / \mathrm{s}$ \\
\hline Wall thickness $h$ & $0.03 \mathrm{~cm}$ & Max flow $\max _{t \in(0, T)}\left(q_{\text {total }}^{\text {inp }}\right)$ & $13.65 \mathrm{~cm}^{3} / \mathrm{s}$ \\
\hline Wall Young's modulus $E$ & $9,863,400$ dyn $/ \mathrm{cm}^{2}$ & Mean inlet pressure $p_{0}$ & $133,333.32 \mathrm{dyn} / \mathrm{cm}^{2}$ \\
\hline Wall Poisson's ratio $\sigma$ & 0.5 & Steady longitudinal velocity $\bar{w}_{s}$ & $22.9 \mathrm{~cm} / \mathrm{s}$ \\
\hline Wall density $\rho^{w}$ & $1 \mathrm{gr} / \mathrm{cm}^{3}$ & Max velocity $\max _{t \in(0, T)}\left(\bar{w}^{\text {inp }}\right)$ & $25.38 \mathrm{~cm} / \mathrm{s}$ \\
\hline Blood density $\rho$ & $1 \mathrm{gr} / \mathrm{cm}^{3}$ & Reynolds number $\rho \bar{w}_{s} 2 R / \mu$ & 343.5 \\
\hline Blood dynamic viscosity $\mu$ & $0.04 \mathrm{poise}$ & Steady pressure gradient $k_{s}$ & $-81.76 \mathrm{dyn} / \mathrm{cm}^{3}$ \\
\hline Time period $T$ & $1.1 \mathrm{~s}$ & Inviscid wave speed $c_{0}$ & $702.26 \mathrm{~cm} / \mathrm{s}$ \\
\hline
\end{tabular}



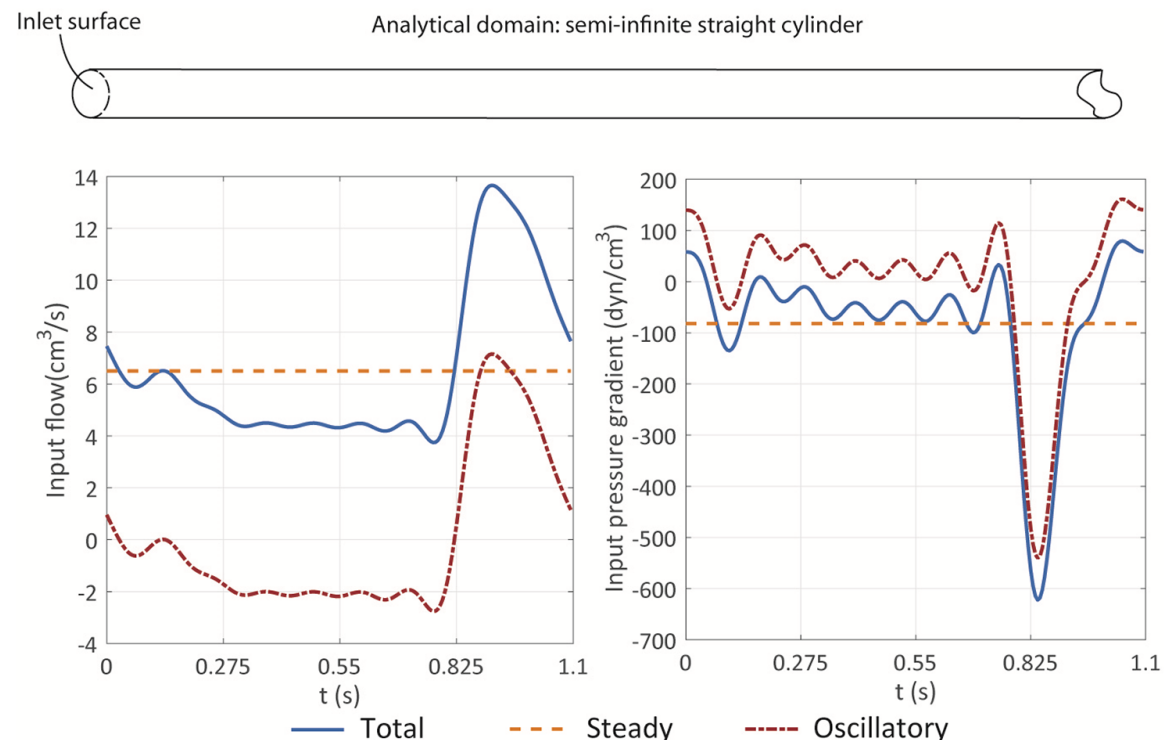

F I G U RE 1 Total input flow (left) and pressure gradient (right), decomposed into steady and oscillatory components.

Analytical domain is shown on the top

T A B LE 4 Fourier coefficients of the reconstructed input flow data, in $\mathrm{cm}^{3} / \mathrm{s}$

\begin{tabular}{llllll}
$\boldsymbol{N}$ & $\mathbf{0}$ & $\mathbf{1}$ & $\mathbf{2}$ & $\mathbf{3}$ & $\mathbf{4}$ \\
$Q_{n}^{\text {inp }}$ & 6.5016 & $2.6735+1.9326 \mathrm{i}$ & $-0.1934+1.9469 \mathrm{i}$ & $-1.4043+0.414 \mathrm{i}$ & $-0.5547-0.5047 \mathrm{i}$ \\
$\boldsymbol{N}$ & 5 & 6 & 7 & 8 & 9 \\
$Q_{n}^{\text {inp }}$ & $0.3293-0.1272 \mathrm{i}$ & $0.17+0.3785 \mathrm{i}$ & $-0.2054+0.1780 \mathrm{i}$ & $-0.0355-0.1522 \mathrm{i}$ & $0.1761-0.0646 \mathrm{i}$ \\
\hline
\end{tabular}

\begin{tabular}{|ll}
\hline Parameters at leading frequency & \\
\hline Womersley numb. $\alpha_{1}$ & 3.585 \\
\hline Real wave speed $c_{R 1}$ & $643.519 \mathrm{~cm} / \mathrm{s}$ \\
\hline Wavelength $\lambda_{1}=T c_{R 1}$ & $707.871 \mathrm{~cm}$ \\
\hline$\delta_{1}$ & 0.0394 \\
\hline$\beta_{1}$ & 0.0027 \\
\hline$\varepsilon_{1}$ & 0.0001 \\
\hline
\end{tabular}

TA B L E 5 Hemodynamic parameters estimated at $\omega_{1}=2 \pi /$ $T \mathrm{rad} / \mathrm{s}$

hemodynamic parameters considered, thus the long-wave and linear approximations are justified. The thin-wall approximation is also satisfied due to small values of $h / R=0.1$ and $\xi(z, t) / R=\delta(z, t) / 2$, Remark 4 .

If we examine the behavior of the parameter $\delta(z, t)=\bar{w}(z, t) / c$ for all frequencies of the imposed inflow waveform, we observe that the absolute value of this parameter remains under $4 \%$ for the entire cycle, further indicating the validity of the linear assumption used in the derivation of the analytical solution.

\subsection{Total analytical solutions in a semi-infinite domain}

This section describes the total analytical solution to demonstrate key spatio-temporal behavior of the wave traveling in a semi-infinite domain $z \in[0, \infty)$. The vessel length is taken to be equal to a spatial wavelength $\lambda_{1}=707.871 \mathrm{~cm}$, over which spatial periodicity and wave attenuation phenomena can be observed.

In Figure 2 we examine the total and oscillatory components of the longitudinal velocity, $w_{\text {total }}$ and $w$, respectively. A typical Womersley velocity profile can be observed in the oscillatory component of the solution, especially at the vessel inlet. Periodicity in time is apparent in the solution. A periodic behavior is also observed in space over the wavelength $\lambda_{1}$. Velocity profiles at $z=0$ and $z=\lambda_{1}$ reveal the same phase, although attenuation is clear in the profiles at $z=\lambda_{1}$. 
F I G URE 2 Longitudinal velocity profiles along the vessel: Periodicity in time with period $T$; periodicity in space with period $\lambda_{1}$ and attenuation in the oscillatory component are observed
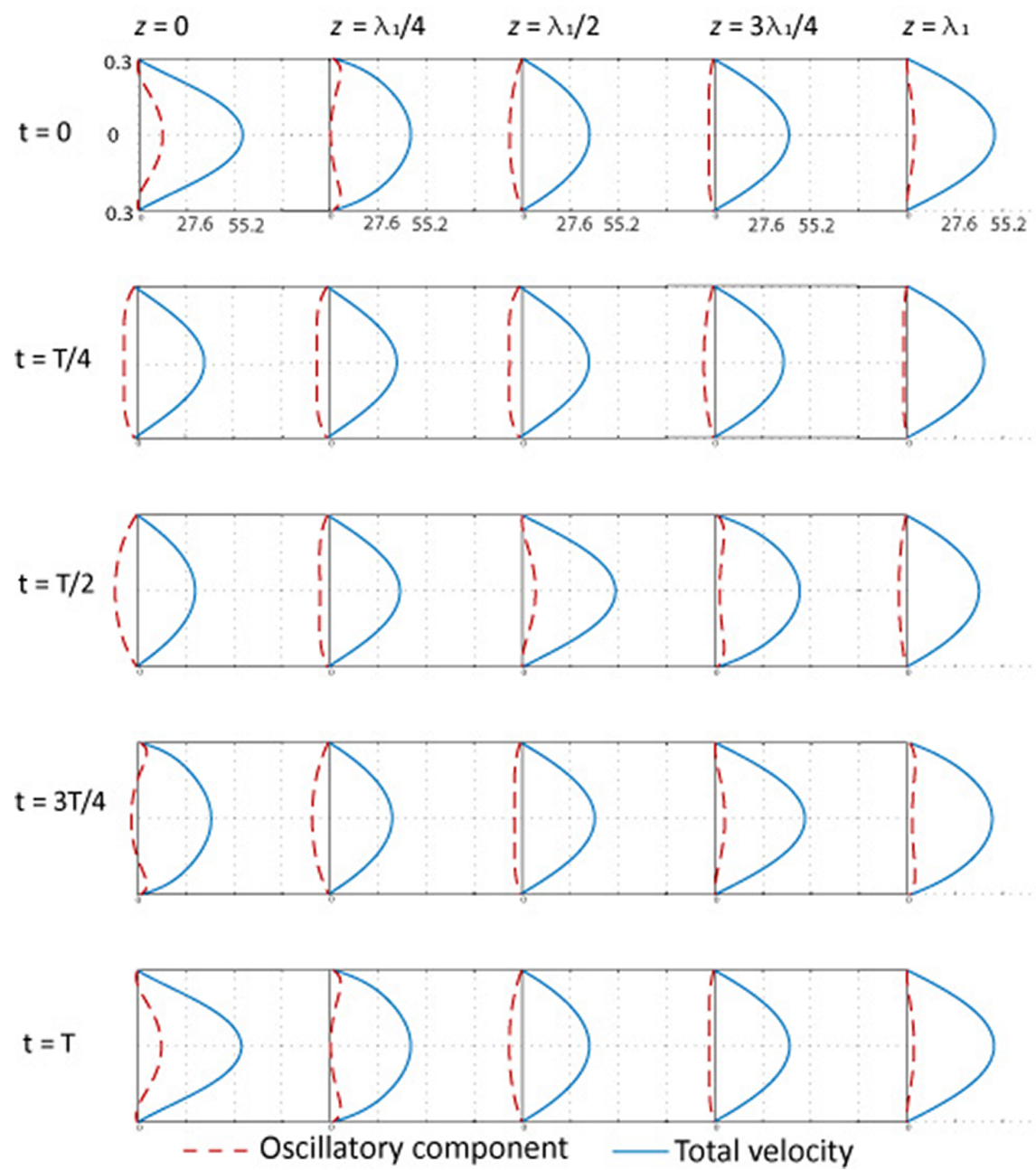

Periodicity in space and attenuation are also demonstrated in the radial and longitudinal components of the wall velocity, shown in Figure 3(a) at different times of the cardiac cycle. Following equations (2) and (3), the wall velocity has only oscillatory component (the steady component is zero). The maximum magnitude of the wall radial velocity is approximately $0.1 \mathrm{~cm} / \mathrm{s}$, much smaller than its longitudinal counterpart (approximately $5 \mathrm{~cm} / \mathrm{s}$ ), and thus consistent with the approximation $\varepsilon / \delta \ll 1$ at the wall.

Figure 3(b) and (c) show the total and oscillatory components of the pressure over the spatial wavelength $\lambda_{1}$ and time, respectively. The constant steady pressure gradient $-k_{s}$ can be observed in the longitudinal distribution of the total pressure at different times of the cardiac cycle, Figure 3(b) (left). Similar to the oscillatory velocity, the oscillatory pressure component gradually attenuates along the vessel, Figure 3 (b) (right). Figure 3(c) depicts the periodic behavior of total and oscillatory pressures at different longitudinal coordinates.

Figure 3(d) shows the total centerline velocity $\left.w_{\text {total }}\right|_{r=0}$ and total pressure over 3 times the leading frequency spatial wavelength, $3 \lambda_{1}$. The plots reveal dissipating oscillations which are almost completely attenuated at the distal end of the vessel. We obtained an exponentially-decaying velocity attenuation curve (gray line in Figure 3 (d), left) as follows: $w_{s}(0)+\max _{z \in\left[0,3 \lambda_{1}\right]}(\operatorname{Re}\{w(0, z, 0)\}) \exp \left(\omega_{1}(z-\hat{z}) / c_{I 1}\right)$, where $\hat{z}$ is the coordinate of the first local maximum at $t=0$.

\section{3 | Verification of numerical solutions in a finite-size vessel}

This section presents an illustrative example of the CMM application and compares numerical results with analytical solutions in a finite-size domain. The same material and hemodynamic parameters from the analytical solution 
(A)

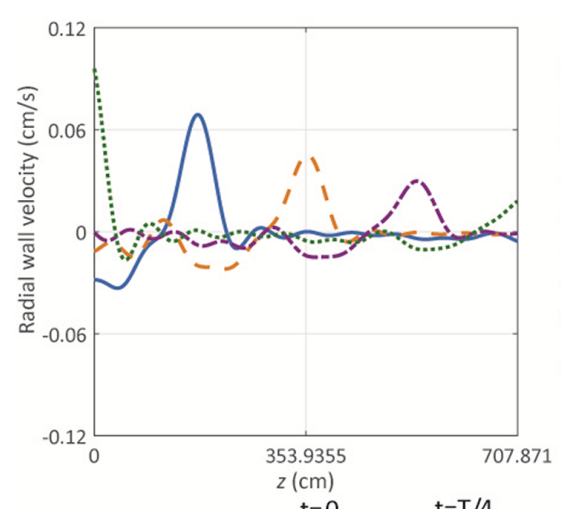

(B)
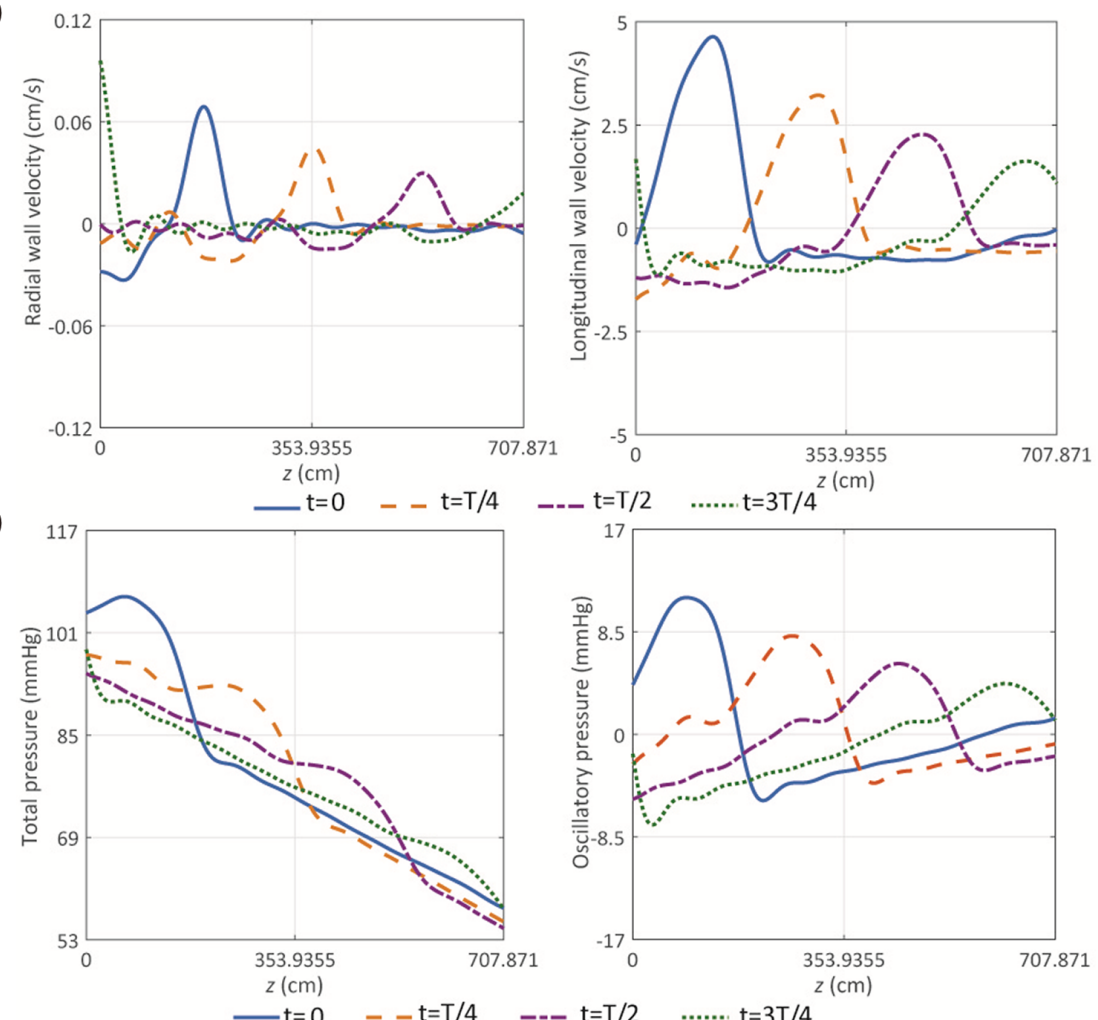

(C)

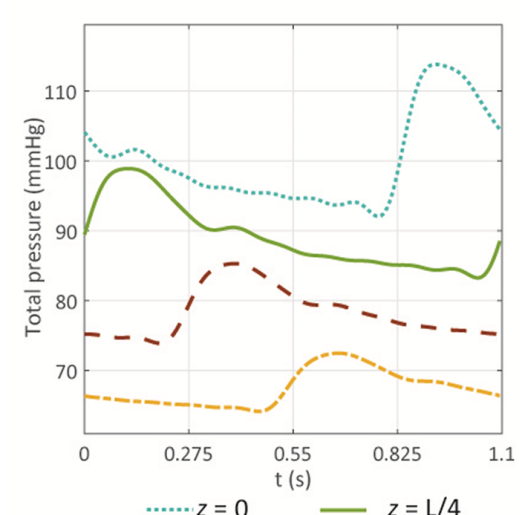

(D)

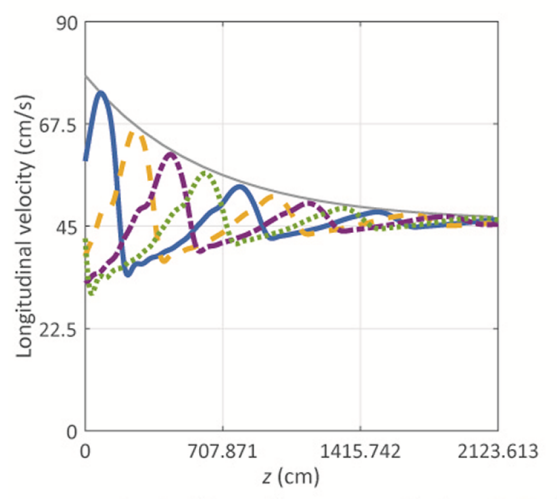

attenuation $-\mathrm{t}=0 \quad-=\mathrm{t}=\mathrm{T} / 4$
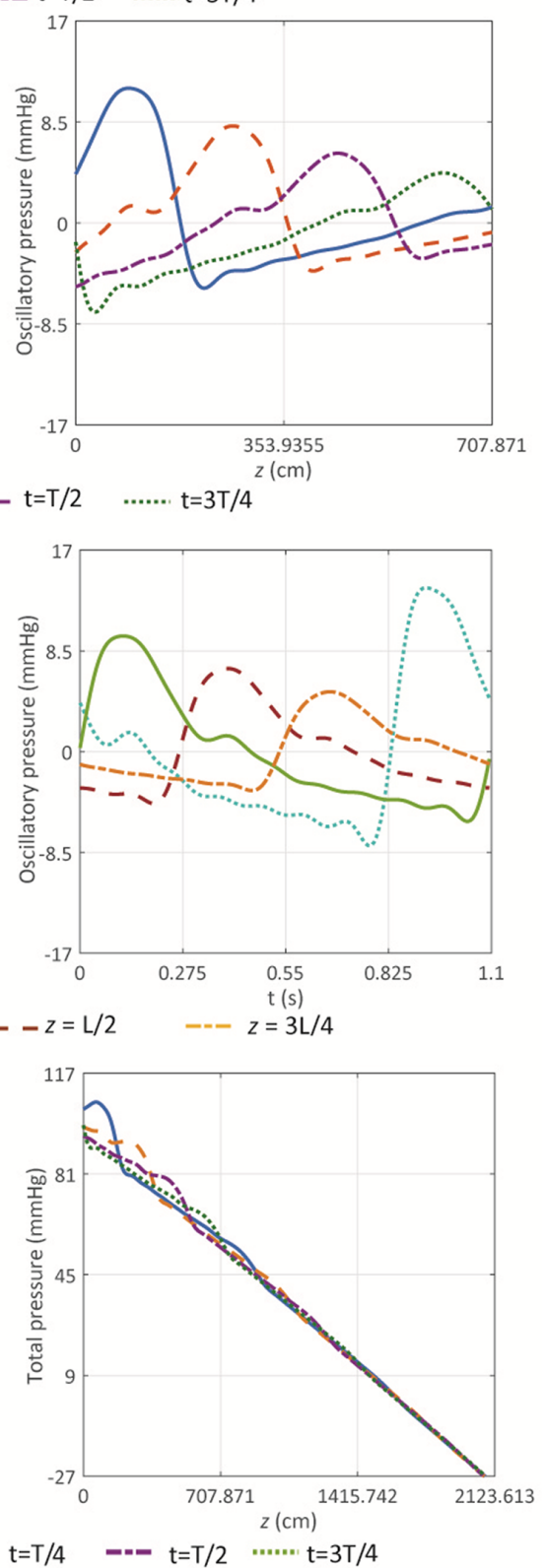

FIGURE 3 (a) Radial and

longitudinal velocity along the vessel wall at different times $m T / 4, m=0, \ldots 3$; (b) Total and oscillatory pressures along the vessel at different times; (c) Total and oscillatory pressure versus time at different cross sections of the vessel $z=m \lambda_{1} / 4, m=0, \ldots 3$; (d) Total centerline velocity and pressure at different times of the cardiac cycle over three spatial wavelengths $L=3 \lambda_{1}$

(Table 3) are considered for the computational CMM solution. A finite vessel length was set to $L=12.6 \mathrm{~cm}$, representing a typical value for the common carotid artery ${ }^{39}$. A transformation of the analytical solution from cylindrical to Cartesian coordinates was adopted in this section. 
FI G URE 4 (a) Inlet and outflow boundary conditions prescribed in the numerical domain: Straight cylinder with a length $L=12.6 \mathrm{~cm}$; (b) cut plane through the central section of the vessel $(z=6.3 \mathrm{~cm})$, showing the structured nature of the mesh in the interior of the vessel

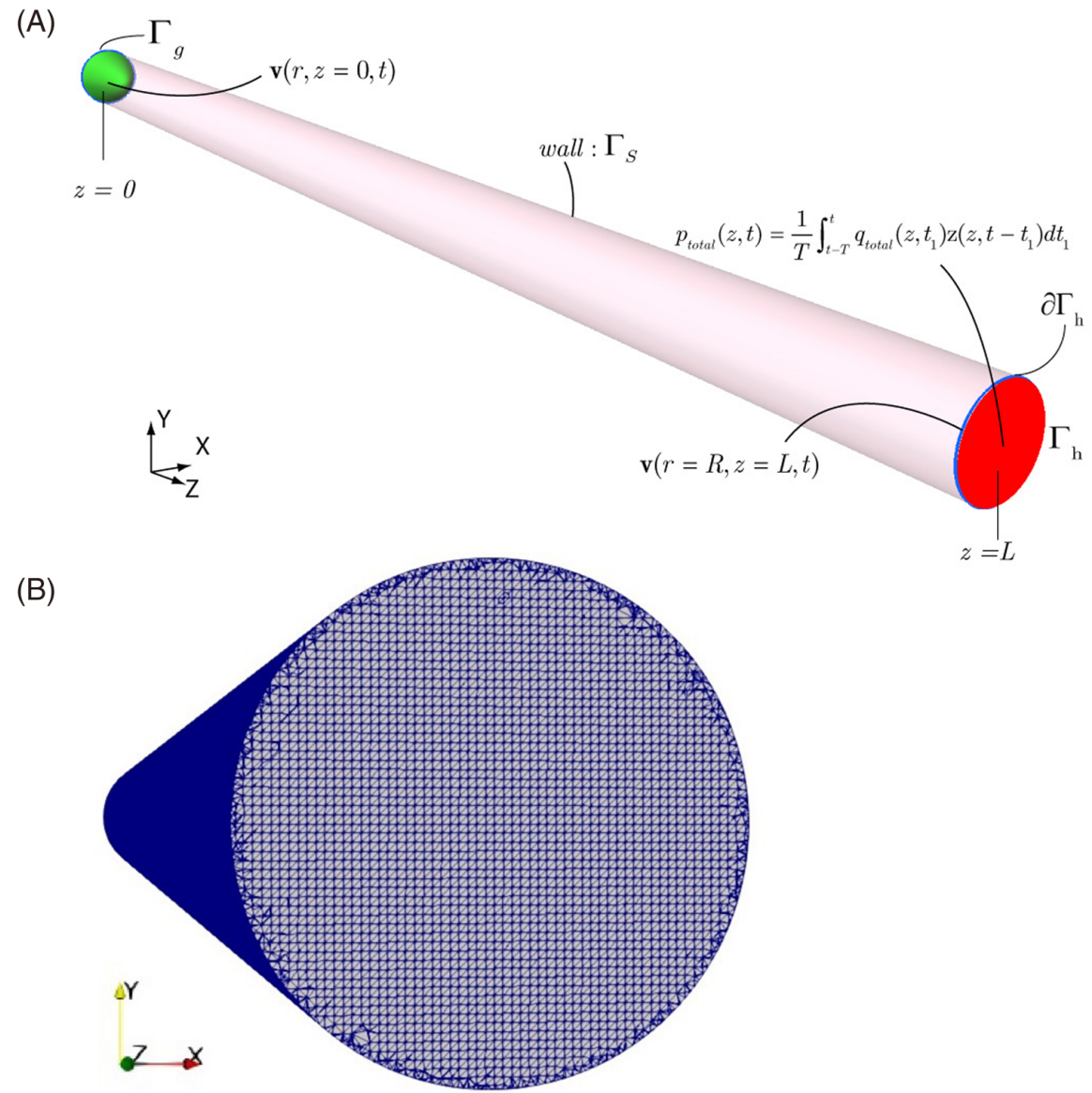

\subsection{1 | Boundary conditions}

Figure 4(a) contains a schematic representation of the boundary conditions of the problem. On the inlet boundary $\bar{\Gamma}_{g}=\Gamma_{g} \cup \partial \Gamma_{g}$, a prescribed velocity field $\mathbf{v}=\left(v_{x}(t), v_{y}(t), v_{z}(t)\right)$ given by Womersley's analytical solution for total velocity at $z=0 \mathrm{~cm}$ is set. On the outflow boundary $\bar{\Gamma}_{\mathrm{h}}=\Gamma_{\mathrm{h}} \cup \partial \Gamma_{\mathrm{h}}$, two conditions are set: i) the impedance boundary condition (27), according to the coupled-multidomain method ${ }^{29}$, is prescribed on the interior nodes of the face $\Gamma_{\mathrm{h}}$ (depicted in red in Figure 4(a)) where the impedance function is defined by equations (18), (19). The numerically integrated flow is filtered to 10 modes to keep consistency with the frequency content of the analytical solution. ii) a prescribed velocity field $\mathbf{v}$ given by Womersley's analytical solution for total wall velocity is set at the boundary wall nodes $\partial \Gamma_{\mathrm{h}}, z=12.6$ $\mathrm{cm}, r=R$ (depicted in blue in Figure 4(a)). Figure 5 shows the 10-term modulus and phase impedance function in the frequency domain $Z_{n}$, as well as its time domain counterpart $\bar{z}(t)$. The vessel wall-fluid interface is $\Gamma_{S}$. Note that no boundary condition is set in the interior of this interface (e.g.) since the solutions for velocity (fluid problem) and wall displacement/velocity (solid problem) are not known a priori and are obtained by solving the CMM formulation.

\subsection{2 | Initial conditions: Steady-state initialization of the CMM}

Initial conditions must be set with the same care used for the boundary condition specification to minimize the impact of initial transients in the system due to lack of equilibrium at the fluid-solid interface. To initialize the problem, we run a steady flow analysis with deformable walls. The following boundary conditions were defined for the steady-state problem:

- Outflow boundary $\Gamma_{\mathrm{h}}$ : we take advantage of knowing the analytical solution for velocity and pressure at $z=L=12.6$ $\mathrm{cm}$ and $t=0 \mathrm{~s}$ to define a resistance boundary condition $R_{\text {out }}$ :

$$
R_{\text {out }}=\frac{p_{\text {total }}(z=L, t=0)}{q_{\text {total }}(z=L, t=0)}=17,152.6 \mathrm{dyn} \cdot \mathrm{s} / \mathrm{cm}^{5} \text { on } \Gamma_{h}
$$


This resistance outflow boundary condition $R_{\text {out }}$ is imposed using a coupled-multidomain formulation ${ }^{29}$ similar to that described in Section 2.2.2.

Inflow boundary $\bar{\Gamma}_{g}$ : a total longitudinal velocity boundary condition at $z=0 \mathrm{~cm}$ and $t=0 \mathrm{~s}$ is imposed, neglecting the radial components of the velocity, viz.

$$
\left.v_{x}(x, y)\right|_{z=0}=\left.v_{y}(x, y)\right|_{z=0}=0,\left.v_{z}(x, y)\right|_{z=0}=\left.w_{\text {total }}(x, y)\right|_{z=0, t=0},(x, y) \text { on } \bar{\Gamma}_{g}
$$

- Outflow boundary wall ring $\partial \Gamma_{\mathrm{h}}$ : the total longitudinal velocity, neglecting the radial component of the velocity, is prescribed:

$$
\left.v_{x}(x, y)\right|_{z=L}=\left.v_{y}(x, y)\right|_{z=L}=0,\left.v_{z}(x, y)\right|_{z=L}=\left.w_{t o t a l}(x, y)\right|_{z=L, t=0},(x, y) \text { on } \partial \Gamma_{\mathrm{h}}
$$

Initial values of pressure and velocity for the steady-state initialization were set to $p_{0}=133,333.32 \mathrm{dyn} / \mathrm{cm}^{2}$ and zero, respectively. Simulations were run for 4,000 time-steps with a time step size of $\Delta t=1.1 \cdot 10^{-5} \mathrm{~s}$, until a converged steady-state solution with momentum residuals smaller than $10^{-4}$ was obtained. This solution provides an optimal initial condition for the pulsatile analysis, since the fluid-solid system is in dynamic equilibrium, and the computed velocity and pressure fields closely match those of the analytical solution at time zero.
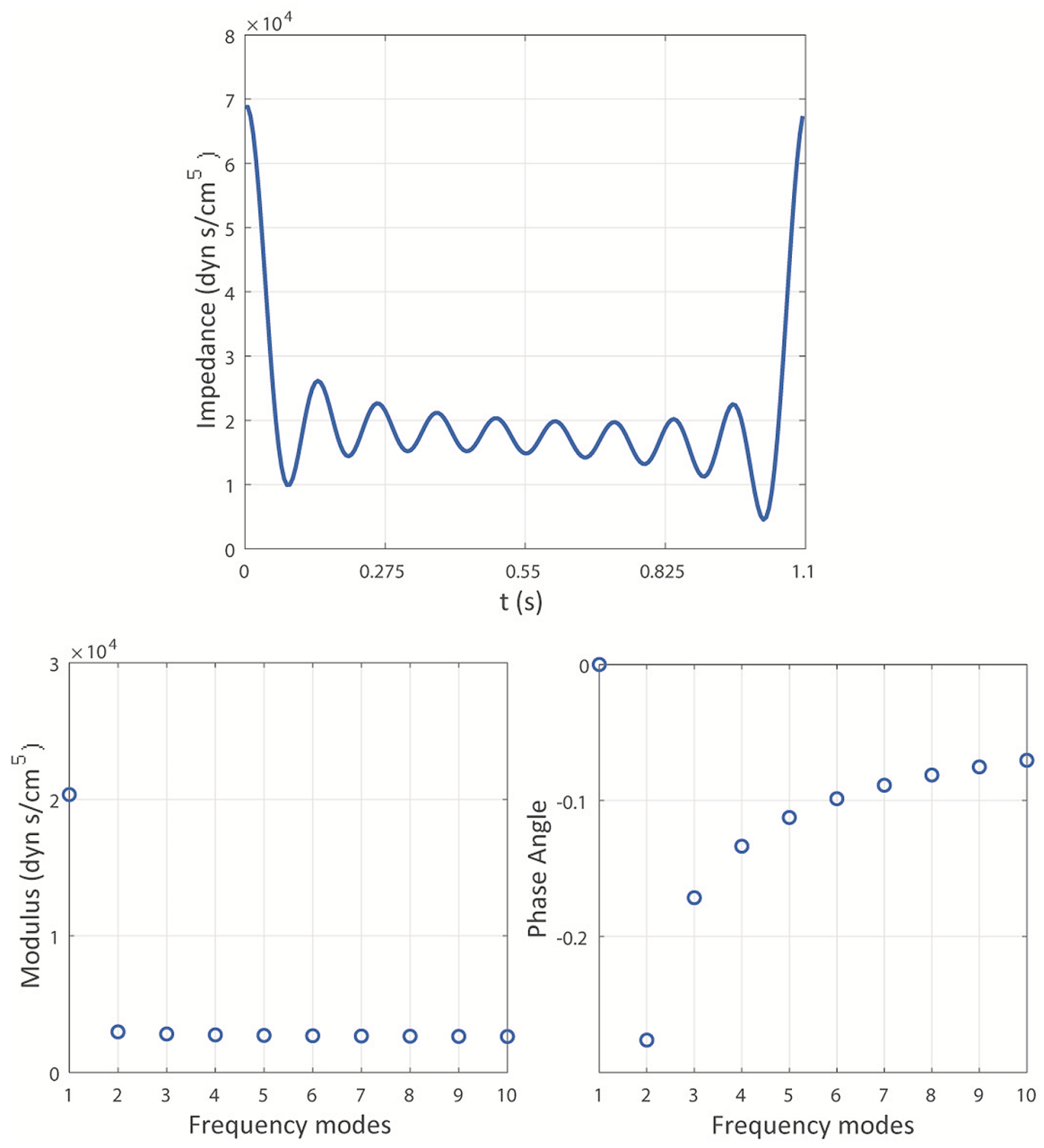

FIG URE 5 Impedance function $z(t)$ in time domain at $z=L=12.6 \mathrm{~cm}$. Inserts depict the 10-mode reconstruction of the modulus and phase of the impedance function in the frequency domain $Z_{\mathrm{n}}$ 
(a)
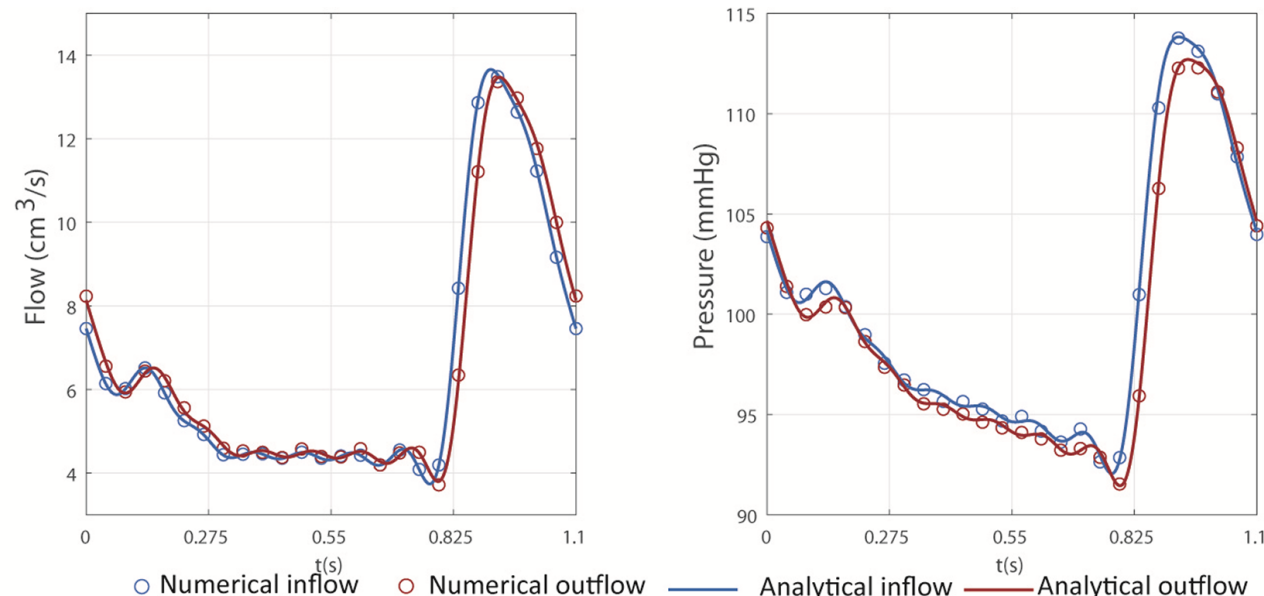

(b)
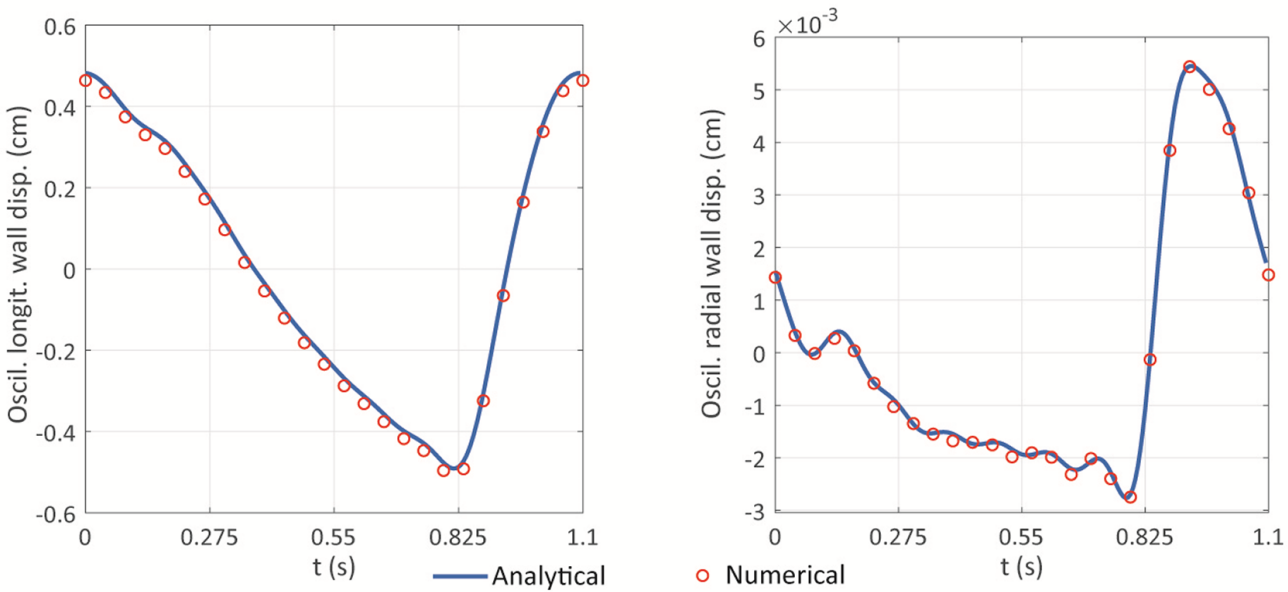

(c)
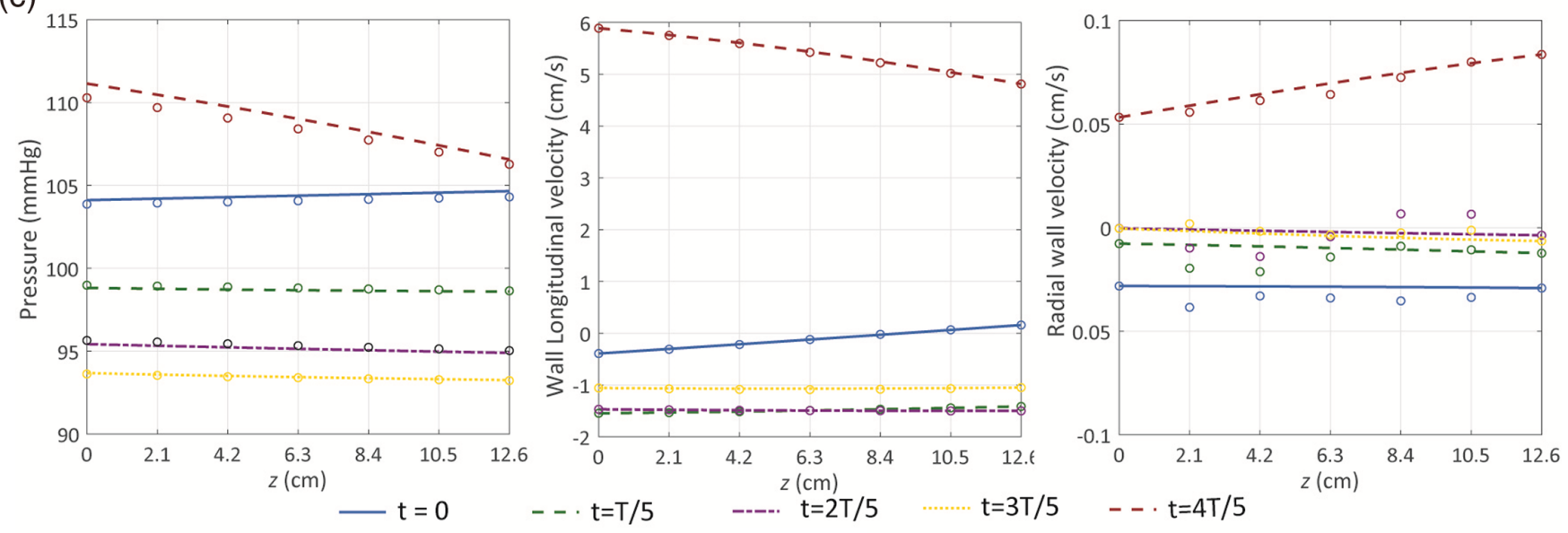

F I G U RE 6 (a) Numerical versus analytical solution: Flow and pressure at inlet and the outlet of the vessel; (b) oscillatory parts of wall longitudinal and radial displacements over time, at $z=L / 2=6.3$; (c) comparison between the analytical (lines) and numerical (circles) wall pressure (left), wall longitudinal velocity (middle), and radial velocity (right) along the vessel axis at different times

\subsubsection{Comparison between numerical (CMM) and analytical (Womersley) solutions}

Numerical simulations were run for three cardiac cycles, using a time step size of $\Delta t=1.1 \cdot 10^{-4} \mathrm{~s}$, and a linear tetrahedral finite element mesh consisting of 3,902,077 nodes and 22,025,114 elements (mesh size $\sim 0.01 \mathrm{~cm}$ ). Figure 4 (b) shows the mesh used in the simulation. It is unstructured in the wall boundary and structured in the interior. 
Longitudinal fluid velocity $(\mathrm{cm} / \mathrm{s})$
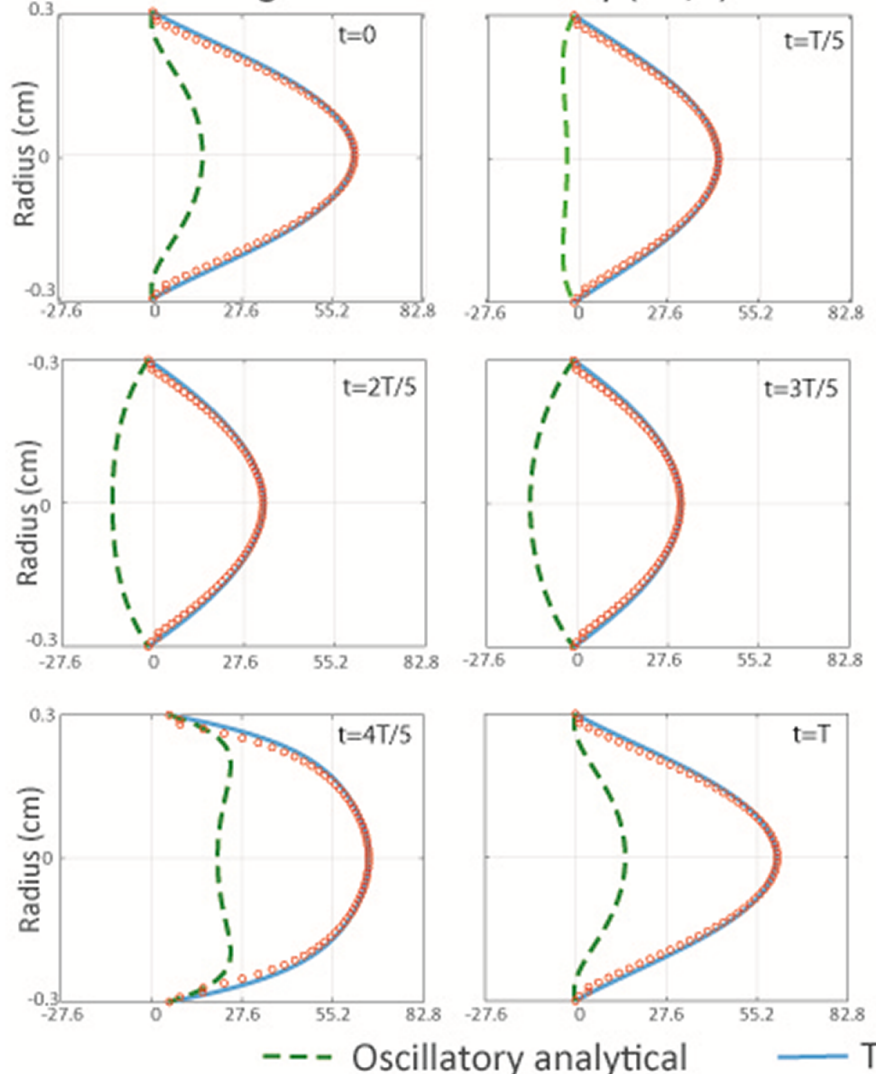

Radial fluid velocity $(\mathrm{cm} / \mathrm{s})$
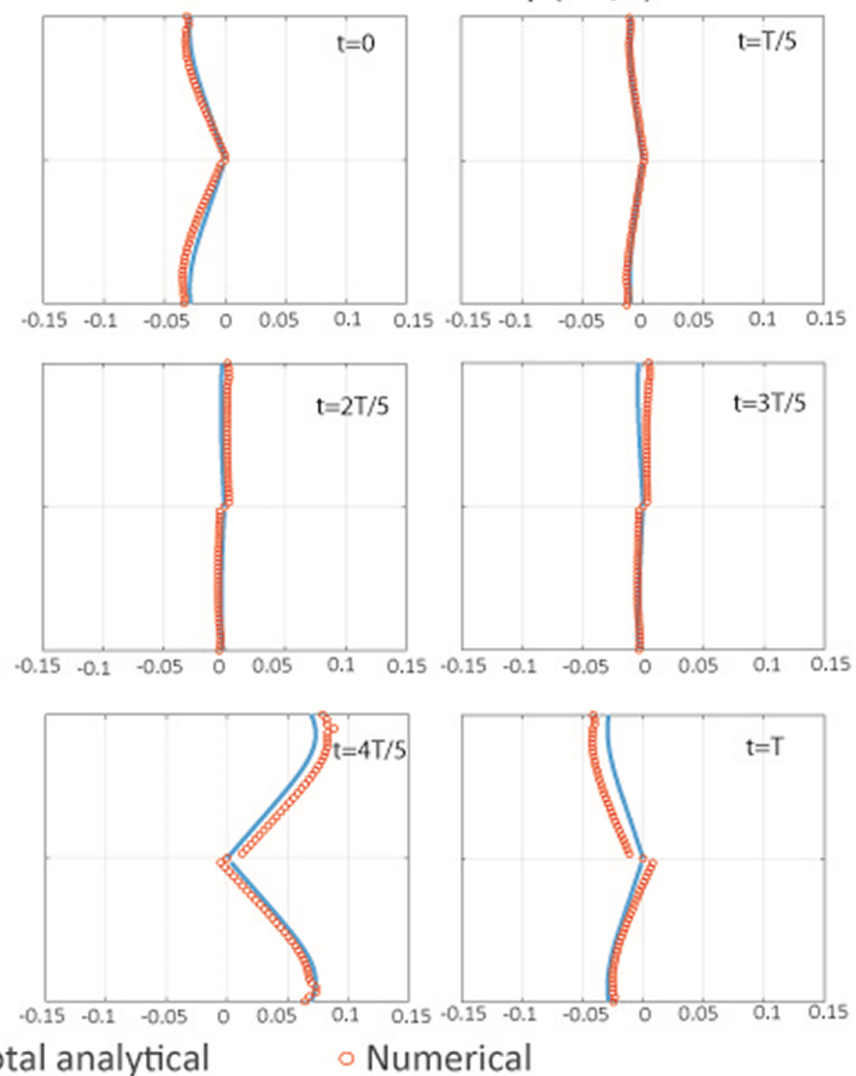

F I G U R E 7 Comparison between analytical and numerical solutions for longitudinal (left) and radial (right) velocity profiles at the central section of the vessel at different times

A structured mesh can better reproduce symmetric patterns, a desirable attribute for comparing the numerical results with the analytical solution.

Flow and pressure waveforms: Figure 6 (a) shows a comparison between analytical and numerical flow and pressure profiles at the inlet and outlet boundaries of the vessel. Pressure waveforms lag flow at both locations, a characteristic trait of hemodynamics in large vessels. The agreement between numerical and analytical solutions is excellent: the relative $\mathrm{L}_{2}$-norm error for outlet flow rate and pressure are $1.06 \%$ and $0.2 \%$, respectively.

Figure 7 shows longitudinal and radial velocity profiles at the central cross-section of the vessel, $z=L / 2=6.3 \mathrm{~cm}$, for different times of the cardiac cycle, for the numerical and analytical solutions. This location was chosen for being the farthest away from the boundaries, and therefore the least subject to the impact from the boundary conditions, which directly prescribe the velocity (inlet face) or impedance function (outlet face). Solutions are plotted along a line $y \in(-R, R)$.

Longitudinal velocities: Figure 7 (left) shows the total analytical velocity, its oscillatory component and the numerical solution. Velocity profiles are shown to be periodic. Wall velocity oscillates around a zero mean, showing negative (backwards) and positive (forward) oscillating values thorough the cycle. The comparison between analytical and numerical total velocity profiles shows a good agreement, with a relative $\mathrm{L}_{2}$-norm error smaller than $6.7 \%$.

Radial velocities: Figure 7 (right) shows a comparison between the analytical and numerical radial velocity profiles. For the fluid domain, the magnitude of the longitudinal velocities is $\sim 30 \mathrm{~cm} / \mathrm{s}$ and the radial velocities $\sim 0.02 \mathrm{~cm} / \mathrm{s}$, a 1,500 ratio. For the wall velocities, this ratio is significantly smaller $\sim 50$, in agreement with Figure 3(a). There is a poor agreement between radial velocity profiles: relative $\mathrm{L}_{2}$-norm error is as large as $178 \%$ for $t=2 T / 5$ and $47 \%$ for $t=T$.

Wall displacements: Figure 6(b) shows a comparison between analytical and numerical oscillatory displacements in the longitudinal (left) and radial (right) directions at $z=L / 2=6.3 \mathrm{~cm}$. The profiles show a good agreement, with relative $\mathrm{L}_{2}$-norm errors in the longitudinal and radial displacements of $5.6 \%$ and $3.8 \%$, respectively. 
Spatial distributions of wall pressure and velocity: Figure 6 (c) shows a comparison between analytical and numerical solutions for wall pressure, longitudinal and radial wall velocities along the vessel at different times. There is a good agreement for the wall pressure and longitudinal velocity (maximum relative $\mathrm{L}_{2}$-norm errors of $0.6 \%$ and $2.2 \%$, respectively). The radial velocity displays good agreement for large velocity values, while for small values the discrepancy increases. This discrepancy is due to the small radial velocity values relative to the main longitudinal components.

Pulse wave propagation: A wave propagation speed can be calculated as the ratio of vessel length and pulse transit time between the inlet and outlet waveforms using the foot-to-foot method ${ }^{40}$. Using the numerical pressure waveforms, a wave speed of $c^{\text {num }}=693 \mathrm{~cm} / \mathrm{s}$ was obtained. This is a $4 \%$ difference from the wave speed obtained using the analytical waveforms $c^{\text {analyt }}=664 \mathrm{~cm} / \mathrm{s}$. By contrast, the Moens-Korteweg formula (Remark 6 in Appendix) produces an estimate for pulse wave velocity in an inviscid fluid of $c_{0}=702.26 \mathrm{~cm} / \mathrm{s}$. This estimate is larger than the previous values because of wave attenuation, present in the numerical and analytical waveforms, but absent in the Moens-Korteweg formula.

Linear behavior and thin wall assumptions: The analytical estimate for the scale parameter $\delta$ defining the magnitude of the contribution of the non-linear advection to the total momentum was found to be smaller than $4 \%$. This bound was confirmed by the numerical solution, which produced an upper bound for the scale parameter $\delta^{\text {num }}=3.6 \%$.

Lastly, the maximum numerical radial wall deformation was $\xi_{\max } / R=0.0186$, indeed small and close to the theoretical estimate of $\delta^{\text {num }} / 2$, equation (17). These values therefore confirm the validity of the linear behavior (used just by Womersley's theory), and the thin wall assumption (used by both Womersley's solution and the CMM).

We supplement our numerical results shown in Figure 6 (b), Figure 7 by movie files in the online version (see Supporting Information). Video 1 demonstrates a 3D profile of the total fluid velocity as it changes over the cardiac cycle (with a scale factor 0.01 ). Video 2 shows $2 \mathrm{D}$ oscillations of the radial fluid velocity (scale factor 2), revealing the lack of axisymmetry. Video 3 and Video 4 demonstrate pulsations of the total wall displacements (scale factor 0.1) and oscillatory radial wall displacements (scale factor 15), respectively.

\section{4 | DISCUSSION AND CONCLUSIONS}

The interaction between fluids and deformable structures is a key component of many multi-physics problems, especially in cardiovascular biomechanics. Modeling pulsatile blood flow within complex deformable vessels requires advanced FSI methods. To ensure credibility of these methods, it is important to perform verification (testing an implementation against an analytical solution) and validation (testing a theoretical model against experimentally acquired data) studies. Even though the CMM had been successfully used for numerous blood flow simulation studies for more than a decade ${ }^{21,39,41}$, including validation against in vitro experimental data ${ }^{42}$, and is a key component of the opensourced software CRIMSON ${ }^{35}$, a rigorous verification study of the method was still lacking.In this paper, we verified the Coupled-Momentum Method FSI method for simulating blood flow in compliant vessels ${ }^{20}$ by comparing it against a Womersley's deformable wall solution ${ }^{10,13}$, which can be regarded as the analytical solution for the CMM under the assumptions of idealized axisymmetric geometry, linear flow and wall responses. A key novelty of this work is the multi-frequency nature of the analytical solution, which allows for accurate representation of cardiovascular flow and pressure waveforms.

A thorough overview of Womersley's analytical solution was first presented. This included a scale analysis to examine the validity of the main assumptions of the theory, namely linear flow and wall dynamics, long-wave and thin-wall approximations. Several non-dimensional parameters were identified: the parameter $\delta=\bar{w} / c<<1$ scales the non-linear fluid inertia terms. For the long-wave approximation to hold, $\beta=\omega R / c \ll 1 . \beta$ scales the viscous stress terms, the pressure radial gradient, and the wall inertia component. The parameter $\varepsilon=\delta \beta$ represents the ratio of a typical radial velocity to the wave speed. Lastly, the vessel wall is subject to the conditions: $\xi / R \ll 1$ (small radial deformations) and $h /$ $R \ll 1$ (thin membrane). Our analysis revealed that $\delta$ is a critical scaling parameter, larger in magnitude than $\beta$ and $\varepsilon$ (Table 5), and proportional to the radial deformation $\xi / R$ (Remark 4). Material parameters for the application examples presented here were chosen such that all the conditions above are valid. In particular, maximum $\delta$ at peak systole $\sim 4 \%$, thus ensuring consistency of the verification.

A verification study of the CMM was then presented. Since Womersley's deformable wall solution is defined over a semi-infinite domain, a key component of this study was to prescribe a reflection-free outflow boundary condition via a characteristic impedance function. From the standpoint of solution verification, this outflow impedance presents 
a 'softer' condition than imposing pressure or velocity. The numerical solutions are therefore 'less constrained', thus enhancing the relevance of the verification study.

The verification study considered an illustrative example of pulsatile blood flow in a straight cylindrical compliant vessel with parameters corresponding to a common carotid artery. Results demonstrated excellent agreement between numerical and analytical solutions for longitudinal velocities, wall displacements, pressure and flow waveforms, and pulse wave velocity. However, large discrepancies were observed between radial velocities. This can be partially explained by their small magnitudes and the small ratio relative to their longitudinal counterparts (a 1/1,500 ratio in fluid velocities, and $1 / 50$ in wall velocities). Another factor potentially contributing to the discrepancy between radial velocity profiles is the lack of 2D axisymmetry of the 3D computational mesh and/or a bias from uniformly oriented structured mesh.

It is well known that the longitudinal component of vessel wall motion is not as large as that predicted by the analytical solution ${ }^{13}$ used here. To address this shortcoming, Womersley incorporated a correction to the theory whereby longitudinal wall motion was constrained via added mass representing the surrounding tissue ${ }^{11}$. Computational FSI techniques can mimic such a constraint by introducing adequate surface traction forces, an approach also developed for the $\mathrm{CMM}^{43}$.

The linear and axisymmetric assumptions of the analytical solution limit the scope of the verification study. Therefore, the CMM verification presented here does not take into account features such as advective inertial forces and complex geometries (noncircular cross-sections, tapering, curvatures, bifurcations, etc.). To address this limitation, in future studies we will compare the CMM against another 3D FSI solvers.

\section{ACKNOWLEDGEMENTS}

This work was supported by the National Institute of Biomedical Imaging and Bioengineering in the National Institute of Health [grant U01 HL135842].

\section{ORCID}

Vasilina Filonova (1) https://orcid.org/0000-0002-0620-3330

Irene E. Vignon-Clementel (10 https://orcid.org/0000-0001-9754-4386

\section{REFERENCES}

1. Milnor WR. Hemodynamics. Williams \& Wilkins; 1989.

2. Morgan GW, Ferrante WR. Wave propagation in elastic tubes filled with streaming liquid. J Acoust Soc am. 1955;27(4):715-725. https:// doi.org/10.1121/1.1908005

3. Witzig K. Uber erzwungene Wellenbewegungen zaher, inkompressibler Flussigkeiten in elastischen Rohren; 1914.

4. Lambossy P. Oscillations forcees d'un liquide incompressibile et visqueux dans un tube rigide et horizontal. Calcul de la force frottement. Helv Phys Acta. 1952;25:371-386.

5. Nichols WW, O'Rourke MF, Vlachopoulos C. McDonald's Blood Flow in Arteries: Theoretical. Hodder Arnold: Experimental and Clinical Principles. Sixth; 2011.

6. Papadakis G. New analytic solutions for wave propagation in flexible, tapered vessels with reference to mammalian arteries. $J$ Fluid Mech. 2011;689:465-488. https://doi.org/10.1017/jfm.2011.424

7. Panasenko GP, Stavre R. Asymptotic analysis of the stokes flow in a thin cylindrical elastic tube. Appl Anal. 2012;91(11):1999-2027. https://doi.org/10.1080/00036811.2011.584187

8. Sazonov I, Nithiarasu P. A novel, FFT-based one-dimensional blood flow solution method for arterial network. Biomech Model Mechanobiol. 2019;18(5):1311-1334. https://doi.org/10.1007/s10237-019-01146-0

9. Womersley JR. Method for the calculation of velocity, rate of flow and viscous drag in arteries when the pressure gradient is known. Annu Rev Fluid Mech. 1955;127:553-563.

10. Womersley JR. An Elastic Tube Theory of Pulse Transmission and Ossilatory Flow in Mammalian Arteries.; 1957.

11. Womersley JR. Oscillatory flow in arteries: the constrained elastic tube as a model of arterial flow and pulse transmission. Phys Med Biol. 1957;2(2):178-187.

12. Womersley JR. Oscillatory flow in arteries. III: flow and pulse-velocity formulae for a liquid whose viscosity varies with frequency. Phys Med Biol. 1958;2(4):374-382. https://doi.org/10.1088/0031-9155/2/4/307

13. Womersley JR. Oscillatory motion of a viscous liquid in a thin-walled elastic tube-I: the linear approximation for long waves. London, Edinburgh, Dublin Philos Mag J Sci. 1955;46(373):199-221. https://doi.org/10.1080/14786440208520564

14. Perktold K, Rappitsch G. Computer simulation of local blood flow and vessel mechanics in a compliant carotid artery bifurcation model. J Biomech. 1995;28(7):845-856. https://doi.org/10.1016/0021-9290(95)95273-8 
15. Gerbeau JF, Vidrascu M, Frey P. Fluid-structure interaction in blood flows on geometries based on medical imaging. Comput Struct. 2005;83(2-3):155-165. https://doi.org/10.1016/j.compstruc.2004.03.083

16. Bazilevs Y, Calo VM, Zhang Y, Hughes TJR. Isogeometric fluid-structure interaction analysis with applications to arterial blood flow. Comput Mech. 2006;38(4-5):310-322. https://doi.org/10.1007/s00466-006-0084-3

17. Peskin CS. Flow patterns around heart valves: a numerical method. J Comput Phys. 1972;10(2):252-271.

18. Peskin CS. The immersed boundary method. Acta Numer. 2002;11(2002):479-517. https://doi.org/10.1017/S0962492902000077

19. Baaijens FPT. A fictitious domain/mortar element method for fluid-structure interaction. Int J Numer Methods Fluids. 2001;35(7):743761. https://doi.org/10.1002/1097-0363(20010415)35:7<743::AID-FLD109>3.0.CO;2-A

20. Figueroa CA, Vignon-Clementel IE, Jansen KE, Hughes TJR, Taylor CA. A coupled momentum method for modeling blood flow in three-dimensional deformable arteries. Comput Methods Appl Mech Eng. 2006;195(41-43):5685-5706. https://doi.org/10.1016/j.cma.2005. 11.011

21. Xiao N, Humphrey JD, Figueroa CA. Multi-scale computational model of three-dimensional hemodynamics within a deformable fullbody arterial network. J Comput Phys. 2013;244:22-40. https://doi.org/10.1016/j.jcp.2012.09.016

22. Hose DR, Lawford PV, Narracott AJ, Penrose JMT, Jones IP. Fluid-solid interaction: benchmarking of an external coupling of ANSYS with CFX for cardiovascular applications. J Med Eng Technol. 2003;27(1):23-31. https://doi.org/10.1080/0309190021000036862

23. Kanyanta V, Ivankovic A, Karac A. Validation of a fluid-structure interaction numerical model for predicting flow transients in arteries. J Biomech. 2009;42(11):1705-1712. https://doi.org/10.1016/j.jbiomech.2009.04.023

24. Ponzini R, Vergara C, Rizzo G, et al. Womersley number-based estimates of blood flow rate in doppler analysis: in vivo validation by means of phase-contrast MRI. IEEE Trans Biomed Eng. 2010;57(7):1807-1815. https://doi.org/10.1109/TBME.2010.2046484

25. van Geel MHA, Giannopapa CG, van der Linden BJ. Development of a Blood Flow Model and Validation against Experimets and Analytical Models. The Netherlands: Eindhoven; 2011.

26. Passerini T, Quaini A, Villa U, Veneziani A, Canic S. Validation of an open source framework for the simulation of blood flow in rigid and deformable vessels. Int J Numer Method Biomed Eng. 2013;29(11):1192-1213. https://doi.org/10.1002/cnm.2568

27. Wylie EB, Streeter VL. Fluid Transients in Systems. Prentice Hall: Englewood Cliffs, New York; 1993.

28. Wiggert DC, Tijsseling AS. Fluid transients and fluid-structure interaction in flexible liquid-filled piping. Appl Mech Rev. 2001;54(5):455481. https://doi.org/10.1115/1.1404122

29. Vignon-Clementel IE, Alberto Figueroa C, Jansen KE, Taylor CA. Outflow boundary conditions for three-dimensional finite element modeling of blood flow and pressure in arteries. Comput Methods Appl Mech Eng. 2006;195(29-32):3776-3796. https://doi.org/10.1016/j. cma.2005.04.014

30. Vignon IE, Taylor CA. Outflow boundary conditions for one-dimensional finite element modeling of blood flow and pressure waves in arteries. Wave Motion. 2004;39(4):361-374. https://doi.org/10.1016/j.wavemoti.2003.12.009

31. Barenblatt GI. Scaling, Self-Similarity, and Intermediate Asymptotics: Dimensional Analysis and Intermediate Asymptotics. Cambridge University Press; 1996.

32. Zamir M. The Physics of Pulsatile Flow. London, Ontario: Springer-Verlag; 2000.

33. Olufsen MS, Peskin CS, Kim WY, Pedersen EM, Nadim A, Larsen J. Numerical simulation and experimental validation of blood flow in arteries with structured-tree outflow conditions. Ann Biomed Eng. 2000;28(11):1281-1299. https://doi.org/10.1114/1.1326031

34. Azer K, Peskin CS. A one-dimensional model of blood flow in arteries with friction and convection based on the Womersley velocity profile. Cardiovasc Eng. 2007;7(2):51-73. https://doi.org/10.1007/s10558-007-9031-y

35. Crimson software. http://www.crimson.software/. Accessed December 18, 2018.

36. Figueroa CA. A coupled-momentum method to model blood flow and vessel deformation in human arteries: applications in disease research amd simulation-based medical planning. 2006;(March).

37. Fung YC. Biomechanics: Circulation. Springer-Verlag; 1997.

38. Pomella N, Wilhelm EN, Kolyva C, González-Alonso J, Rakobowchuk M, Khir AW. Common carotid artery diameter, blood flow velocity and wave intensity responses at rest and during exercise in young healthy humans: a reproducibility study. Ultrasound Med Biol. 2017;43(5):943-957. https://doi.org/10.1016/j.ultrasmedbio.2016.12.018

39. Vignon-Clementel IE, Figueroa CA, Jansen KE, Taylor CA. Outflow boundary conditions for 3D simulations of non-periodic blood flow and pressure fields in deformable arteries. Comput Methods Biomech Biomed Engin. 2010;13(5):625-640. https://doi.org/10.1080/ 10255840903413565

40. Gaddum NR, Alastruey J, Beerbaum P, Chowienczyk P, Schaeffter T. A technical assessment of pulse wave velocity algorithms applied to non-invasive arterial waveforms. Ann Biomed Eng. 2013;41(12):2617-2629. https://doi.org/10.1007/s10439-013-0854-y

41. Taylor CA, Figueroa CA. Patient-specific modeling of cardiovascular mechanics. Annu Rev Biomed Eng. 2009;11(1):109-134. https://doi. org/10.1146/annurev.bioeng.10.061807.160521

42. Kung E, Baretta A, Baker C, et al. Predictive modeling of the virtual hemi-Fontan operation for second stage single ventricle palliation: two patient-specific cases. J Biomech. 2013;46(2):423-429. https://doi.org/10.1016/j.jbiomech.2012.10.023

43. Moireau P, Xiao N, Astorino M, et al. External tissue support and fluid-structure simulation in blood flows. Biomech Model Mechanobiol. 2012;11(1-2):1-18. https://doi.org/10.1007/s10237-011-0289-z 


\section{SUPPORTING INFORMATION}

Additional supporting information may be found online in the Supporting Information section at the end of this article.

How to cite this article: Filonova V, Arthurs CJ, Vignon-Clementel IE, Figueroa CA. Verification of the coupled-momentum method with Womersley's Deformable Wall analytical solution. Int J Numer Meth Biomed Engng. 2019;36:e3266. https://doi.org/10.1002/cnm.3266

\section{APPENDIX}

Derivation of the deformable wall Womersley's solution for a linear system of governing equations, Table 1, is described next.

\section{Harmonic waves}

Solutions to the linear system of governing equations can be obtained via superposition of harmonic waves. Separation of variables is assumed for each unknown, as well as periodicity in time with frequency $\omega$ :

$$
\begin{gathered}
u^{\prime}=u_{1}^{\prime}\left(r^{\prime}\right) \exp \left(i\left(t^{\prime}-z^{\prime}\right)\right), w^{\prime}=w_{1}^{\prime}\left(r^{\prime}\right) \exp \left(i\left(t^{\prime}-z^{\prime}\right)\right), p^{\prime}=p_{1}^{\prime}\left(r^{\prime}\right) \exp \left(i\left(t^{\prime}-z^{\prime}\right)\right) \\
\xi^{\prime}=K^{\prime} \exp \left(i\left(t^{\prime}-z^{\prime}\right)\right), \zeta^{\prime}=N^{\prime} \exp \left(i\left(t^{\prime}-z^{\prime}\right)\right)
\end{gathered}
$$

Here, $K^{\prime}, N^{\prime}$ are non-dimensional constants, independent of $r^{\prime}$, due to the fixed mean radial displacement assumption (equation (12)). For convenience, the non-dimensional variable $\eta=\Lambda r / R=\Lambda r^{\prime}$ and parameter $\Lambda=i^{3 / 2} \alpha$ are introduced. Substituting $u_{1}^{\prime}, w_{1}^{\prime}, p_{1}^{\prime}$ from equation (A1) into the fluid governing equations, Table $1(\mathrm{~A})$, expressing variables in terms of $\eta$ and using $i \alpha^{2}=-\Lambda^{2}$, we obtain:

$$
\begin{gathered}
\eta \frac{d u_{1}^{\prime}(\eta)}{d \eta}+u_{1}^{\prime}(\eta)-\eta \frac{i}{\Lambda} w_{1}^{\prime}(\eta)=0 \\
\eta^{2} \frac{d^{2} u_{1}^{\prime}(\eta)}{d \eta^{2}}+\eta \frac{d u_{1}^{\prime}(\eta)}{d \eta}+\left(\eta^{2}-1\right) u_{1}^{\prime}(\eta)=i \Lambda \eta^{2} \frac{\gamma}{\beta^{2}} \frac{d p_{1}^{\prime}(\eta)}{d \eta} \\
\eta \frac{d^{2} w_{1}^{\prime}(\eta)}{d \eta^{2}}+\frac{d w_{1}^{\prime}(\eta)}{d \eta}+\eta w_{1}^{\prime}(\eta)=\gamma \eta p_{1}^{\prime}(\eta) .
\end{gathered}
$$

Equations (A4) and (A5) are Bessel differential equations of first and zero order, respectively, and can be solved in closed-form for $u_{1}^{\prime}$ and $w_{1}^{\prime}$ as a function of the pressure $p_{1}^{\prime}$. The continuity equation (A3) can then be used to obtain the pressure. Applying the finite-velocity condition at the vessel centerline $\eta=0$, Table $1(\mathrm{C})$, the solutions to the fluid momentum equations that satisfy the continuity equation given in terms of Bessel functions of the first kind $J_{n}$ become:

$$
\begin{aligned}
& u_{1}^{\prime}(\eta)=i \frac{G^{\prime}}{\Lambda} J_{1}(\eta)-i \gamma H^{\prime} \frac{c R}{\nu\left(i^{3} \alpha^{2}-\chi^{2}\right)} J_{1}\left(\frac{\chi}{\Lambda} \eta\right), \\
& w_{1}^{\prime}(\eta)=G^{\prime} J_{0}(\eta)-\gamma H^{\prime} \frac{c R \chi}{\nu\left(i^{3} \alpha^{2}-\chi^{2}\right)} J_{0}\left(\frac{\chi}{\Lambda} \eta\right), \\
& p_{1}^{\prime}(\eta)=H^{\prime} J_{0}\left(\frac{\chi}{\Lambda} \eta\right),
\end{aligned}
$$


where $G^{\prime}, H^{\prime}$ are constants and $\chi=i \beta$. These solutions can be simplified further by noting that $\chi \ll 1$, and thus: $\left(i^{3} \alpha^{2}-\chi^{2}\right)^{-1}=1 / i^{3} \alpha^{2}+O\left(\chi^{2}\right) \approx-1 / i \alpha^{2}$. In addition, properties of Bessel functions yield: $J_{0}(\chi \eta / \Lambda) \approx 1, J_{1}(\chi \eta / \Lambda) \approx 0.5 \chi \eta /$ $\Lambda$. Thus, $p_{1}^{\prime} \approx H^{\prime}, \partial p_{1}^{\prime} / \partial \eta \approx-H^{\prime} \chi \eta / 2 \Lambda=-H^{\prime} \chi r^{\prime} / 2$, which implies that pressure is near constant over the cross section of the vessel (e.g., radial pressure gradient is small but not zero). Using these simplifications, the solutions given by equations (A6) become:

$$
\begin{gathered}
u_{1}^{\prime}\left(r^{\prime}\right)=i \frac{G^{\prime}}{\Lambda} J_{1}\left(\Lambda r^{\prime}\right)+i \frac{\gamma}{2} H^{\prime} r^{\prime}, \\
w_{1}^{\prime}\left(r^{\prime}\right)=G^{\prime} J_{0}\left(\Lambda r^{\prime}\right)+\gamma H^{\prime}, \\
p_{1}^{\prime}=H^{\prime} .
\end{gathered}
$$

At this point, the non-dimensional constants $H^{\prime}, G^{\prime}, K^{\prime}, N^{\prime}$ must be determined. This is accomplished by: 1) substituting equations (A1), (A2), (A8), (A9) to Table 1(B); and 2) applying the kinematic boundary condition at the fluid-solid interface, Table $1(\mathrm{C})$, to produce the following algebraic system:

$$
\begin{aligned}
& \gamma H^{\prime}-v K^{\prime}+i \sigma v N^{\prime}=0, \\
& g J_{0}(\Lambda) G^{\prime}+2 \sigma v K^{\prime}+i 2\left(\frac{\rho^{s} h}{\rho R}-v\right) N^{\prime}=0, \\
& \gamma H^{\prime}+g J_{0}(\Lambda) G^{\prime}-2 K^{\prime}=0, \\
& \gamma H^{\prime}+J_{0}(\Lambda) G^{\prime}-i N^{\prime}=0,
\end{aligned}
$$

with $v=B h / \rho R c^{2}$ and $g=2 J_{1}(\Lambda) / \Lambda J_{0}(\Lambda)$. The above is a homogeneous system depending on the wave speed $c$. Therefore a solution for $c$ ensuring a zero determinant must be obtained to find a non-trivial solution for $H^{\prime}, G^{\prime}, K^{\prime}, N^{\prime}$.

\section{Frequency dependent wave speed}

Enforcing the determinant of the system of equations (A10) to be zero produces the following quadratic equation:

$$
\left[(1-g)\left(1-\sigma^{2}\right)\right] v^{2}-\left[2+\frac{\rho^{w} h}{\rho R}(1-g)+g\left(\frac{1}{2}-2 \sigma\right)\right] v+g+2 \frac{\rho^{w} h}{\rho R}=0
$$

known as frequency equation, and whose solution provides an expression for the wave speed $c$ as a function of the material properties of the fluid and wall, as well as the Womersley number $\alpha . c$ is a complex number and therefore is not a true speed in the physical sense. The complex wave speed can be decomposed into its real $c_{R}=\left(\operatorname{Re}\left\{c^{-1}\right\}\right)^{-1}$ and imaginary $c_{I}=\left(\operatorname{Im}\left\{c^{-1}\right\}\right)^{-1}$ parts, and thus the exponential expression for the phase variation becomes:

$$
\exp (i \omega(t-z / c))=\exp \left(\omega z / c_{I}\right) \exp \left(i \omega\left(t-z / c_{R}\right)\right)
$$

The imaginary part of the wave speed $c_{I}$ effectively changes the amplitude of the waves, thus representing an attenuation effect. The real part of the wave speed $c_{R}$ effectively changes the phase of the wave, adopting different values for different frequencies, thus representing a dispersion effect.

Remark 6 It is common in the clinical research community to use the Moens-Korteweg formula to relate pulse wave velocity of blood flow with the structural stiffness of the vessel: $c_{0}^{2}=E h / 2 \rho R$. However, this wave speed $c_{0}$ is for a perfect (inviscid) fluid, while blood is viscous. If viscosity is taken into account, the wave speed is no longer frequency-independent. Womersley showed that the variation in pulse velocity with frequency and viscosity can be expressed as a function of the non-dimensional Womersley number $\alpha^{10}$. From the definition of the parameters $v$ and $B$, it follows that the wave speed $c$ can be written as a function of the inviscid wave speed $c_{0}$ as: Unlike $c, c_{0}$ is a real quantity. Therefore, attenuation is absent in inviscid fluids since $\operatorname{Im}\left\{c_{0}\right\}=0$. Also the complex parameter $v$ is 
proportional to square of wave speeds ratio, $v \propto c_{0}^{2} / c^{2}$, and when $v=2 /\left(1-\sigma^{2}\right)$ the wave speed is $c=c_{R}=c_{0}$.

$$
c=c_{0} \sqrt{2 / v\left(1-\sigma^{2}\right)} .
$$

\section{Analytical solution}

Once the wave speed $c$ is obtained, to define the solution to the rank-three system (40), the value for one of the four constants $\left(H^{\prime}, G^{\prime}, K^{\prime}, N^{\prime}\right)$ must be set. Equation (A9) suggests $H^{\prime}$ as a natural choice. $H^{\prime}$ represents the prescribed amplitude of the inlet oscillatory pressure. The remaining constants $G^{\prime}, K^{\prime}, N^{\prime}$ become:

$$
G^{\prime}=-\gamma M H^{\prime} / J_{0}(\Lambda), K^{\prime}=\gamma H^{\prime}(1-M g) / 2, N^{\prime}=i \gamma H^{\prime}(M-1) .
$$

Here $M=(2+v(2 \sigma-1)) / v(2 \sigma-g)$ is the 'elasticity factor', dependent on frequency and wave speed $c$. Then from equations (A2), (A7), (A8) and (A14), the radial and longitudinal components of flow velocity and wall displacements are obtained:

$$
\begin{aligned}
& u^{\prime}\left(r^{\prime}, z^{\prime}, t^{\prime}\right)=\frac{i \gamma H^{\prime}}{2}\left(r^{\prime}-M \frac{2 J_{1}\left(\Lambda r^{\prime}\right)}{\Lambda J_{0}(\Lambda)}\right) \exp \left(i\left(t^{\prime}-z^{\prime}\right)\right), \\
& w^{\prime}\left(r^{\prime}, z^{\prime}, t^{\prime}\right)=\gamma H^{\prime}\left(1-M \frac{J_{0}\left(\Lambda r^{\prime}\right)}{J_{0}(\Lambda)}\right) \exp \left(i\left(t^{\prime}-z^{\prime}\right)\right), \\
& \xi^{\prime}\left(z^{\prime}, t^{\prime}\right)=\frac{\gamma H^{\prime}}{2}(1-M g) \exp \left(i\left(t^{\prime}-z^{\prime}\right)\right), \\
& \zeta^{\prime}\left(z^{\prime}, t^{\prime}\right)=i \gamma H^{\prime}(M-1) \exp \left(i\left(t^{\prime}-z^{\prime}\right)\right) .
\end{aligned}
$$

At the vessel inlet, the dimensional pressure constant is $H=\rho \delta c^{2} \gamma H^{\prime}$. The flow rate is computed from the longitudinal velocity as:

$$
q(z, t)=2 \pi \int_{0}^{R} r w(r, z, t) d r=\pi R^{2} \bar{w}(z, t)=Q \exp (i \omega(t-z / c)), Q=\frac{\pi R^{2} H}{\rho c}(1-M g)
$$

Constants $H$ and $Q$ represent the imposed oscillatory pressure or flow, respectively. Applying equations (5), (6), the dimensional solutions of the system (A16) at fixed frequency $\omega$ are obtained, see Table 2. Since solutions are complex, only the real parts of velocities, pressure and displacements are taken.

\section{Multiple-frequency solution}

Multi-frequency forms are needed for representing physiologically realistic cardiovascular waveforms. Assuming periodicity, waveforms can be represented using the Discrete Fourier transform:

$$
f(z, t)=\sum_{n=-N / 2}^{N / 2} F\left(z, \omega_{n}\right) \exp \left(i \omega_{n} t\right), \quad F\left(z, \omega_{n}\right)=\frac{1}{T} \int_{-T / 2}^{T / 2} f(z, t) \exp \left(-i \omega_{n} t\right) d t,
$$

where $f(z, t)=q(z, t), p(z, t)$ are real functions in the time domain with Fourier coefficients $F\left(z, \omega_{n}\right)=Q_{n}\left(z, \omega_{n}\right), P_{n}\left(z, \omega_{n}\right)$; $\omega_{n}=2 \pi n / T, n=-N / 2, \ldots, N / 2$, and $N$ is the number of Fourier modes. The same transform can be applied to each component of velocity or wall displacement in Womersley's solution.

For each individual frequency $\omega_{n}$, the Womersley's solution for pressure and flow is, for $n \neq 0$ :

$$
P_{n}\left(z, \omega_{n}\right)=H_{n} \exp \left(-i \omega_{n} z / c\right), Q_{n}\left(z, \omega_{n}\right)=\frac{\pi R^{2} H_{n}}{\rho c_{n}}\left(1-M_{n} g_{n}\right) \exp \left(-i \omega_{n} z / c\right) .
$$

For $n=0$, the steady-state solution is used. Therefore, the total (oscillatory plus steady) pressure and flow solutions are: 


$$
\begin{aligned}
& p_{\text {total }}(z, t)=p_{0}+k_{s} z+2 \operatorname{Re}\left\{\sum_{n=1}^{N / 2} P_{n} \exp \left(i \omega_{n} t\right)\right\}, \\
& q_{\text {total }}(z, t)=-\frac{k_{s} \pi R^{4}}{8 \mu}+2 \operatorname{Re}\left\{\sum_{n=1}^{N / 2} Q_{n} \exp \left(i \omega_{n} t\right)\right\},
\end{aligned}
$$

where only positive modes are used given that $p_{\text {total }}$ and $q_{\text {total }}$ are real and thus $P_{n}\left(z, \omega_{-n}\right)=P_{n}\left(\bar{z}, \omega_{n}\right)$ and $Q_{n}\left(z, \omega_{-n}\right)=Q_{n}\left(\bar{z}, \omega_{n}\right)$. 